\title{
Topological models for stable motivic invariants of regular number rings
}

\author{
Tom Bachmann ${ }^{\left(\mathbb{D}_{1}\right.}$ and Paul Arne $\emptyset_{\text {stvær }}{ }^{(\mathbb{D}}{ }_{2}$ \\ ${ }^{1}$ Department of Mathematics, LMU Munich; E-mail: tom.bachmann@zoho.com. \\ ${ }^{2}$ Department of Mathematics F. Enriques, University of Milan, Italy; E-mail: paul.oestvaer@unimi.it \& \\ Department of Mathematics, University of Oslo, Norway; E-mail: paularne@math.uio.no.
}

Received: 22 February 2021; Revised: 28 September 2021; Accepted: 3 November 2021

2020 Mathematics Subject Classification: Primary - 14F35, 14F42, 19E15, 55P42

\begin{abstract}
For an infinity of number rings we express stable motivic invariants in terms of topological data determined by the complex numbers, the real numbers and finite fields. We use this to extend Morel's identification of the endomorphism ring of the motivic sphere with the Grothendieck-Witt ring of quadratic forms to deeper base schemes.
\end{abstract}

\section{Contents}

1 Introduction 1

2 Nilpotent completions $\quad 4$

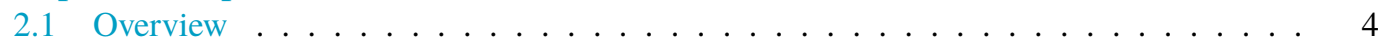

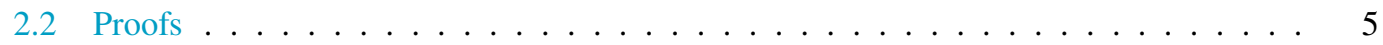

3 Rigidity for stable motivic homotopy of henselian local schemes 9

4 Topological models for stable motivic homotopy of regular number rings 12

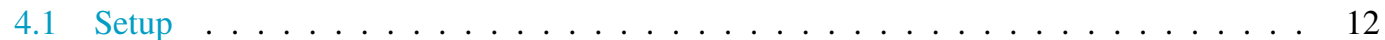

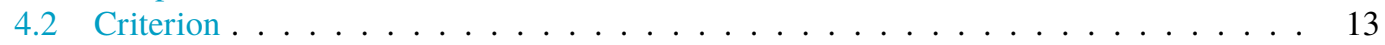

4.3 Models for stable motivic homotopy types . . . . . . . . . . . . . . 16

5 Applications to slice completeness and universal motivic invariants 22

\section{Introduction}

The mathematical framework for motivic homotopy theory has been established over the last 25 years [47]. An interesting aspect witnessed by the complex and real numbers, $\mathbb{C}$, $\mathbb{R}$, is that Betti realisation functors provide mutual beneficial connections between the motivic theory and the corresponding classical and $C_{2}$-equivariant stable homotopy theories [46], [29], [33], [14], [26], [39], [40]. We amplify this philosophy by extending it to deeper base schemes of arithmetic interest. This allows us to understand the fabric of the cellular part of the stable motivic homotopy category of $\mathbb{Z}[1 / 2]$ in terms of $\mathbb{C}, \mathbb{R}$ and $\mathbb{F}_{3}$ - the field with three elements. If $\ell$ is a regular prime, a number theoretic notion introduced by Kummer in 1850 to prove certain cases of Fermat's last theorem [73], we show an analogous result for the ring $\mathbb{Z}[1 / \ell]$.

(C) The Author(s), 2022. Published by Cambridge University Press. This is an Open Access article, distributed under the terms of the Creative Commons Attribution licence (https://creativecommons.org/licenses/by/4.0/), which permits unrestricted re-use, distribution, and reproduction in any medium, provided the original work is properly cited. 
For context, recall that a scheme $X$ - for example, an affine scheme $\operatorname{Spec}(A)$ - has an associated pro-space $X_{e ́ t}$, denoted by $A_{e ́ t}$ in the affine case, called the étale homotopy type of $X$ representing the étale cohomology of $X$ with coefficients in local systems; see [3] and [27] for original accounts and [38, $\S 5]$ for a modern definition. For specific schemes, $X_{e ́ t}$ admits an explicit description after some further localisation; see the work of Dwyer-Friedlander in [23, 24]. For example, they established the pushout square

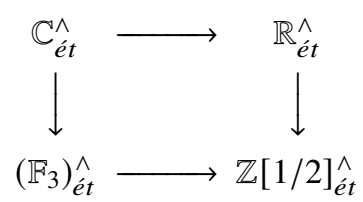

Here the completion $(-)^{\wedge}$ takes into account the cohomology of the local coefficient systems $\mathbb{Z} / 2^{n}(m)$.

Remark 1.1. If $k$ is a field, then $k_{e ́ t}$ is a pro-space of type $K(\pi, 1)$, where $\pi$ is the Galois group over $k$ of the separable closure of $k$. If $S$ is a henselian local ring with residue class field $k$, then $k_{\text {ét }} \rightarrow S_{\text {ét }}$ is an equivalence (by Galois descent, this reduces to the case $S$ strictly henselian local, which is clear). For instance, $\mathbb{C}_{e ́ t} \simeq *$ is contractible, $\mathbb{R}_{e ́ t} \simeq \mathbb{R P}^{\infty}$ is equivalent to the classifying space of the group $C_{2}$ of order 2 and $\left(\mathbb{F}_{p}\right)_{e ́ t} \simeq\left(\mathbb{Z}_{p}\right)_{e ́ t}$ is equivalent to the profinite completion of a circle. That is, up to completion, (1.1) can be expressed more suggestively as $\mathbb{Z}[1 / 2]_{e ́ t} \simeq S^{1} \vee \mathbb{R} \mathbb{P}^{\infty}$. For our generalisation to stable motivic homotopy invariants, it will be essential to keep track of the fields and not just their étale homotopy types.

The presentation of $\mathbb{Z}[1 / 2]_{\hat{e} t}^{\wedge}$ has powerful consequences; for example, taking the 2-adic étale $K$-theory of (1.1) yields a pullback square. Combined with the Quillen-Lichtenbaum conjecture for the 2-primary algebraic $K$-theory of $\mathbb{Z}[1 / 2]$ (see [17], [74], [58], [34]), one obtains the pullback square

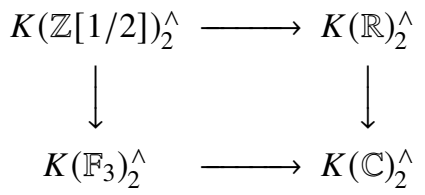

We show that replacing algebraic $K$-theory in (1.2) by an arbitrary cellular motivic spectrum over $\mathbb{Z}[1 / 2]$ still yields a pullback square. Let $\mathcal{S H}(X)$ denote the motivic stable homotopy category of $X$; see [42], [22], [54, §5], [11, §4.1]. We write $\mathcal{S H}(X)^{\text {cell }} \subset \mathcal{S H}(X)$ for the full subcategory of cellular motivic spectra [20]; that is, the localising subcategory generated by the bigraded spheres $S^{p, q}$ for all integers $p, q \in \mathbb{Z}$. For simplicity we state a special case of Theorem 4.7; see Example 4.10.

Theorem 1.2. For every $\mathcal{E} \in \mathcal{S H}(\mathbb{Z}[1 / 2])^{\text {cell }}$ there is a pullback square

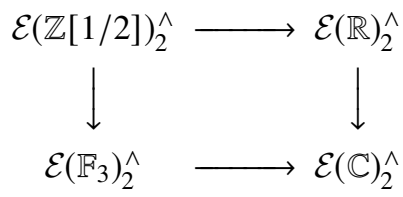

Here, for $X \in \operatorname{Sch}_{\mathbb{Z}[1 / 2]}$, we denote by $\mathcal{E}(X)$ the (ordinary) spectrum of maps from $\mathbf{1}_{X}$ to $p^{*} \mathcal{E}$ in $\mathcal{S H}(X)$, where $\mathbf{1}_{X} \in \mathcal{S H}(X)$ denotes the unit object and $p: X \rightarrow \mathbb{Z}[1 / 2]$ is the structure map.

Example 1.3. The motivic spectra representing algebraic $K$-theory, KGL, hermitian $K$-theory, KO, Witt-theory, KW, motivic cohomology or higher Chow groups, HZ, and algebraic cobordism, MGL, are cellular (at least after localisation at 2) by respectively [20, Theorem 6.2], [62, Theorem 1], [36, Proposition 8.1] and [69, Corollary 10.4], [20, Theorem 6.4]. We refer to [10, Proposition 8.12] for cellularity of the corresponding (very effective or connective) covers $\mathrm{kgl}, \mathrm{ko}, \mathrm{kw}$, in the sense of [70] and Milnor-Witt motivic cohomology $\mathrm{H} \widetilde{\mathbb{Z}}$, in the sense of [8], [6]. 
In the case of $\mathcal{E}=\mathrm{KGL}$, Theorem 1.2 recovers the stable version of [34, Theorem 1.1], and for $\mathcal{E}=\mathrm{KO}$ it recovers [15, Theorem 1.1] (in fact, we extend these results to arbitrary 2-regular number fields, not necessarily totally real). The squares for $\mathrm{KW}, \mathrm{HZ}, \mathrm{H} \widetilde{Z}, \mathrm{MGL}, \mathrm{kgl}$, ko, kw appear to be new.

A striking application of Theorem 1.2 is that it relates the universal motivic invariants over $\mathbb{Z}[1 / 2]$ to the same invariants over $\mathbb{C}, \mathbb{R}$ and $\mathbb{F}_{3}$. That is, applying (1.3) to the motivic sphere $\mathcal{E}=\mathbf{1}_{\mathbb{Z}[1 / 2]}$ enables computations of the stable motivic homotopy groups of $\mathbb{Z}[1 / 2]$. We identify, up to odd-primary torsion, the endomorphism ring of $\mathbf{1}_{\mathbb{Z}[1 / 2]}$ with the Grothendieck-Witt ring of quadratic forms of the Dedekind domain $\mathbb{Z}[1 / 2]$ defined in [53, Chapter IV, §3]. This extends Morel's fundamental computation of $\pi_{0,0}(\mathbf{1})$ over fields $[54, \S 6]$ to an arithmetic situation.

Theorem 1.4. The unit map $\mathbf{1}_{\mathbb{Z}[1 / 2]} \rightarrow \mathrm{KO}_{\mathbb{Z}[1 / 2]}$ induces an isomorphism

$$
\pi_{0,0}\left(\mathbf{1}_{\mathbb{Z}[1 / 2]}\right) \otimes \mathbb{Z}_{(2)} \cong \mathrm{GW}(\mathbb{Z}[1 / 2]) \otimes \mathbb{Z}_{(2)}
$$

Remark 1.5. The étale homotopy types of various other rings and applications to algebraic $K$-theory and group homology of general linear groups were worked out in [23], [24], [57], [34]. We show similar generalisations of (1.3) with $\mathbb{Z}[1 / 2]$ replaced by $\mathcal{O}_{F}[1 / 2]$, for $F$ any 2-regular number field, or by $\mathbb{Z}[1 / \ell], \mathbb{Z}\left[1 / \ell, \zeta_{\ell}\right]$, where $\ell$ is an odd regular prime and $\zeta_{\ell}$ is a primitive $\ell$ th root of unity; to achieve this, we slightly alter the other terms in (1.3). See Theorems 4.7, 4.11, 4.14, 5.2 for precise statements.

Another application, which will be explored elsewhere, is the spherical Quillen-Lichtenbaum property saying the canonical map from stable motivic homotopy groups to stable étale motivic homotopy groups is an isomorphism in certain degrees. Slice completeness is an essential input for showing the spherical property; we deduce this for base schemes such as $\mathbb{Z}[1 / 2]$ in Proposition 11.

As a final comment, we expect that most of the applications we establish hold over more general base schemes, where convenient reductions to small fields are not possible. The proofs will require significantly different ideas.

\section{Organisation}

In Section 2 we give proofs for some more or less standard facts about nilpotent completions in stable $\infty$-categories with $t$-structures. While these results are relatively straightforward generalisations of Bousfield's pioneering work [18], we could not locate a reference in the required generality. These nilpotent completions will be our primary tool throughout the rest of the article. In Section 3 we prove a variant of Gabber rigidity. We show that, for example, if $E \in \mathcal{S H}(X)^{\text {cell }}$ where $X$ is essentially smooth over a Dedekind scheme, then $E\left(X_{x}^{h}\right)_{\ell}^{\wedge} \simeq E(x)_{\ell}^{\wedge}$ for any point $x \in X$ such that $\ell$ is invertible in $k(x)$. Here $X_{x}^{h}$ denotes the henselisation of $X$ along $x$. Our principal results are shown in Section 4 . We establish a general method for exhibiting squares as above and provide a criterion for cartesianess in terms of étale and real étale cohomology; see Proposition 7. Next we verify this criterion for regular number rings, reducing essentially to global class field theory - which is also how Dwyer-Friedlander established (1.1). In Section 5 we discuss some applications, including a proof of Theorem 1.4.

\section{Notation and conventions}

We freely use the language of (stable) infinity categories, as set out in [48, 49]. Given a (stable) $\infty$-category $\mathcal{C}$ and objects $c, d \in \mathcal{C}$, we denote by $\operatorname{Map}(c, d)=\operatorname{Map}_{\mathcal{C}}(c, d)$ (respectively $\operatorname{map}(c, d)=$ $\operatorname{map}_{\mathcal{C}}(c, d)$ ) the mapping space (respectively mapping spectrum). Given a symmetric monoidal category $\mathcal{C}$, we denote the unit object by $\mathbf{1}=\mathbf{1}_{\mathcal{C}}$. We assume familiarity with the motivic stable category $\mathcal{S H}(S)$; see, for example, [11, §4.1]. We write $\Sigma^{p, q}=\Sigma^{p-q} \wedge \mathbb{G}_{m}^{\wedge q}$ for the bigraded suspension functor and $S^{p, q}=\Sigma^{p, q} \mathbf{1}$ for the bigraded spheres. 


\section{Nilpotent completions}

We axiomatise some well-known facts about nilpotent completions in presentably symmetric monoidal stable $\infty$-categories with a $t$-structure. Our arguments are straightforward generalisations of [18] and [50]. Theorems 2.1 and 2.2 are the main results in this section.

\subsection{Overview}

Throughout we let $\mathcal{C}$ be a presentably symmetric monoidal $\infty$-category (i.e., the tensor product preserves colimits in each variable separately) provided with a $t$-structure which is compatible with the symmetric monoidal structure (i.e., $\mathcal{C}_{\geq 0} \otimes \mathcal{C}_{\geq 0} \subset \mathcal{C}_{\geq 0}$ ) and weakly left complete, by which we mean that for $X \in \mathcal{C}$ we have $X \simeq \lim _{n} X_{\leq n}$. Given $E \in \operatorname{CAlg}(\mathcal{C})$ and $X \in \mathcal{C}$, recall [51, Construction 2.7] the standard cosimplicial resolution (or cobar construction)

$$
\Delta_{+} \rightarrow \mathcal{C},[n] \mapsto X \otimes E^{\otimes n+1}
$$

whose limit is (for us by definition) the E-nilpotent completion $X_{E}^{\wedge}$.

We call $X \in \mathcal{C}$ bounded below if $X \in \cup_{n} \mathcal{C}_{\geq n}$. Recall that $R \in \operatorname{CAlg}\left(\mathcal{C}^{\odot}\right)$ is called idempotent if the multiplication map $R \otimes R \rightarrow R \in \mathcal{C}^{\ominus}$ is an equivalence.

Theorem 2.1. Let $\mathcal{C}$ be weakly left complete, $E \in \operatorname{CA} \lg \left(\mathcal{C}_{\geq 0}\right)$ and $X \in \mathcal{C}$. Suppose that $\pi_{0} E \in \operatorname{CAlg}\left(\mathcal{C}^{\diamond}\right)$ is idempotent and $X$ is bounded below. Then the canonical map

$$
X_{E}^{\wedge} \rightarrow X_{\pi_{0} E}^{\wedge}
$$

is an equivalence.

One way of producing idempotent algebras is by taking quotients of the unit. Given $L_{1}, \ldots, L_{n} \in \mathcal{C}_{\geq 0}$ and maps $x_{i}: L_{i} \rightarrow \mathbf{1}$, we set

$$
X /\left(x_{1}^{m_{1}}, x_{2}^{m_{2}}, \ldots, x_{n}^{m_{n}}\right)=X \otimes \operatorname{cof}\left(x_{n}^{\otimes m_{n}}: L_{n}^{\otimes m_{n}} \rightarrow 1\right) \otimes \cdots \otimes \operatorname{cof}\left(x_{1}^{\otimes m_{1}}: L_{1}^{\otimes m_{1}} \rightarrow 1\right) .
$$

The object $\pi_{0}\left(\mathbf{1} /\left(x_{1}, \ldots, x_{n}\right)\right) \in \operatorname{CA} \lg \left(\mathcal{C}^{\odot}\right)$ is idempotent. For varying $m$, the $\mathbf{1} / x_{i}^{m} \mathrm{~s}$ form an inverse system indexed on $\mathbb{N}$ in an evident way; by taking tensor products, the objects $X /\left(x_{1}^{m_{1}}, \ldots, x_{n}^{m_{n}}\right)$ form an $\mathbb{N}^{n}$-indexed inverse system. We define the $x$-completion of $X$ as the limit

$$
X_{x_{1}, \ldots, x_{n}}^{\wedge}:=\lim _{m_{1}, \ldots, m_{n}} X /\left(x_{1}^{m_{1}}, \ldots, x_{n}^{m_{n}}\right) .
$$

Theorem 2.2. Suppose each $L_{i} \in \mathcal{C}_{\geq 0}$ is strongly dualisable with dual $D L_{i} \in \mathcal{C}_{\geq 0}$. If $X \in \mathcal{C}$ is bounded below and $\mathcal{C}$ is weakly left complete, then there is a canonical equivalence

$$
X_{\pi_{0}\left(1 /\left(x_{1}, \ldots, x_{n}\right)\right)}^{\wedge} \simeq X_{x_{1}, \ldots, x_{n}}^{\wedge}
$$

To apply Theorem 2.2 in motivic stable homotopy theory we consider, for a scheme $S$, the homotopy $t$-structure on $\mathcal{S H}(S)$; see, for example, [11, §B], [66, §1].

Theorem 2.3. Let $S$ be a noetherian scheme of finite Krull dimension and suppose $X \in \mathcal{S H}(S)$ is bounded below.

1. There is an equivalence $X_{\mathrm{MGL}}^{\wedge} \simeq X_{\eta}^{\wedge}$.

2. If $1 / \ell \in S$, then there is an equivalence $X_{H \mathbb{F}_{\ell}}^{\wedge} \simeq X_{\eta, \ell}^{\wedge}$. 
Proof. The homotopy $t$-structure is weakly left complete by [66, Corollary 3.8].

(1) Owing to [36, Theorem 3.8, Corollary 3.9] we have MGL $\in \mathcal{S H}(S)_{\geq 0}$ and $\pi_{0}$ (MGL) $\simeq \pi_{0}(\mathbf{1} / \eta)$.

(2) We need to prove that $\mathrm{HF}_{\ell} \in \mathcal{S H}(S)_{\geq 0}$ and $\pi_{0}\left(\mathrm{HF}_{\ell}\right) \simeq \pi_{0}(\mathbf{1} /(\eta, \ell))$. Since $x_{i} \in \pi_{2 i, i} \mathrm{MGL}$ and $\Sigma^{2 i, i} \mathrm{MGL}=\Sigma^{i} \mathbb{G}_{m}^{\wedge i} \wedge \mathrm{MGL} \in \mathcal{S H}(S)_{\geq i} \subset \mathcal{S H}(S)_{>0}$, both of these claims follow from the HopkinsMorel isomorphism

$$
\mathrm{HF}_{\ell} \simeq \mathrm{MGL} /\left(\ell, x_{1}, x_{2}, \ldots\right)
$$

shown in [69, Theorem 10.3]. ${ }^{1}$

Remark 2.4. Theorem 2.3 implies that a map $\alpha: E \rightarrow F \in \mathcal{S H}(S)_{\geq 0}$ is an $(\eta, \ell)$-adic equivalence if and only if $\alpha \wedge \mathrm{HF}_{\ell}$ is an equivalence, which is also easily seen by considering homotopy objects. This weaker statement, however, cannot be used as a replacement for Theorem 2.3 in this work.

\subsection{Proofs}

Recall that $\mathcal{C}$ is a presentably symmetric monoidal $\infty$-category equipped with a compatible $t$-structure.

\section{Definition 1.}

1. Let $E \in \mathrm{CAlg}(\mathcal{C})$. Then $X \in \mathcal{C}$ is $E$-nilpotent if it lies in the thick subcategory generated by objects of the form $E \otimes Y$ for $Y \in \mathcal{C}$.

2. Let $R \in \operatorname{CAlg}\left(\mathcal{C}^{\diamond}\right)$ be idempotent. Then $F \in \mathcal{C}^{\diamond}$ is strongly $R$-nilpotent if $F$ admits a finite filtration whose subquotients are $R$-modules. ${ }^{2}$ Moreover, $X \in \mathcal{C}$ is strongly $R$-nilpotent if it is bounded in the $t$-structure and all homotopy objects are strongly $R$-nilpotent.

Example 2.5. If $X \in \mathcal{C}$ is an $E$-module in the homotopy category, then it is a summand of $X \otimes E$ and thus $X$ is $E$-nilpotent.

Lemma 2.6. Suppose $R \in \operatorname{CAlg}\left(\mathcal{C}^{\odot}\right)$ is idempotent.

1. Let

$$
A \rightarrow B \rightarrow C \rightarrow D \rightarrow E \in \mathcal{C}^{\diamond}
$$

be an exact sequence. If $A, B, D, E$ are strongly $R$-nilpotent, then so is $\mathcal{C}$.

2. An object $X \in \mathcal{C}$ is strongly $R$-nilpotent if and only if it is $R$-nilpotent and bounded in the $t$-structure.

Proof. (1) The proofs of [50, Lemmas 7.2.7-7.2.9] apply unchanged. (2) Example 2.5 implies that strongly $R$-nilpotent objects are $R$-nilpotent, being finite extensions of homotopy $R$-modules. It thus suffices to show that if $X$ is $R$-nilpotent, then its homotopy objects $\pi_{i}^{\mathcal{C}}(X) \in \mathcal{C}^{\odot}$ are strongly $R$-nilpotent. This is clear for free $R$-modules, and the property is preserved by taking summands and shifts and cofibres by (1). The result follows.

\section{Definition 2.}

1. If $E \in \operatorname{CAlg}(\mathcal{C}), X \in \mathcal{C}$, a tower of the form

$$
X \rightarrow \cdots \rightarrow X_{2} \rightarrow X_{1} \rightarrow X_{0}
$$

is called an E-nilpotent resolution if each $X_{i}$ is $E$-nilpotent and for every $E$-nilpotent $Y \in \mathcal{C}$, we have

$$
\underset{n}{\operatorname{colim}}\left[X_{n}, Y\right] \stackrel{\simeq}{\rightarrow}[X, Y] .
$$

${ }^{1}$ This reference assumes $S$ noetherian, but since the equivalence exists over $\mathbb{Z}[1 / \ell]$ it persists after pullback to $S$.

${ }^{2}$ Note that $R$ being idempotent is a property, not additional data. 
2. If $R \in \operatorname{CAlg}\left(\mathcal{C}^{\ominus}\right)$ is idempotent and $X \in \mathcal{C}$, a tower of the form

$$
X \rightarrow \cdots \rightarrow X_{2} \rightarrow X_{1} \rightarrow X_{0}
$$

is called a strongly $R$-nilpotent resolution if each $X_{i}$ is strongly $R$-nilpotent and for every strongly $R$-nilpotent $Y \in \mathcal{C}$, we have

$$
\underset{n}{\operatorname{colim}}\left[X_{n}, Y\right] \stackrel{\simeq}{\rightarrow}[X, Y]
$$

Proposition 3. For $X, Y \in \mathcal{C}$ and $X_{\bullet}, Y_{\bullet}$ E-nilpotent (respectively strongly $R$-nilpotent) resolutions, we have

$$
\operatorname{Map}_{\operatorname{Pro}(\mathcal{C})}\left(X_{\bullet}, Y_{\bullet}\right) \simeq \lim _{n} \operatorname{Map}\left(X, Y_{\bullet}\right)
$$

Thus, any map $X \rightarrow Y$ induces a canonical morphism of towers $X_{\bullet} \rightarrow Y_{\bullet}$. In particular, if $X \simeq Y$, then $X_{\bullet} \simeq Y_{\bullet} \in \operatorname{Pro}(\mathcal{C})$ and $\lim _{n} X_{n} \simeq \lim _{n} Y_{n}$.

Proof. Essentially, by definition we have

$$
\operatorname{Map}\left(X_{\bullet}, Y_{\bullet}\right) \simeq \lim _{n} \operatorname{colim}_{m} \operatorname{Map}\left(X_{m}, Y_{n}\right)
$$

The colimit is equivalent to $\operatorname{Map}\left(X, Y_{n}\right)$ by the definition of a resolution.

Lemma 2.7. Let $E \in \mathrm{CAlg}(\mathcal{C})$ and $X \in \mathcal{C}$.

1. The tower of partial totalisations of the standard cosimplicial objects $X \otimes E^{\otimes \bullet}$ is an E-nilpotent resolution of $X$.

2. Suppose that $E \in \mathcal{C}_{\geq 0}$ and $\pi_{0} E$ is idempotent. Then if $X \rightarrow X_{\bullet}$ is any E-nilpotent resolution by bounded below objects (e.g., if $X$ is bounded below, the one arising from (1)), then $X \rightarrow \tau_{\leq \bullet} X_{\bullet}$ is a strongly $\pi_{0}(E)$-nilpotent resolution.

Proof. (1) Since partial totalisations are finite limits, they commute with $\otimes X$, by stability, and are thus given by $X_{i}=X \otimes \operatorname{cof}\left(I^{\otimes i} \rightarrow \mathbf{1}\right)$, where $I=\operatorname{fib}(\mathbf{1} \rightarrow E)$, see [51, Proposition 2.14]. In the notation of loc. cit. we get $\operatorname{cof}\left(X_{i} \rightarrow X_{i-1}\right) \simeq \Sigma \operatorname{cof}\left(T_{i}(E, X) \rightarrow T_{i-1}(E, X)\right)$ and $X_{0}=0$. This implies $X_{i}$ is $E$-nilpotent by [51, Proposition 2.5(1)]. To conclude, it suffices to prove that if $Y$ is $E$-nilpotent, then $\operatorname{colim}_{i} \operatorname{map}\left(X_{i}, Y\right) \simeq \operatorname{map}(X, Y)$. The class of objects $Y$ satisfying the latter equivalence is thick, so we may assume that $Y$ is an $E$-module. We are reduced to proving that $\operatorname{colim}_{i} \operatorname{map}\left(I^{\otimes i} \otimes X, Y\right)=0$. But this is a summand of $\operatorname{colim}_{i} \operatorname{map}\left(I^{\otimes i} \otimes X \otimes E, Y\right), Y$ being an $E$-module, and the transition maps $I^{\otimes i+1} \otimes E \rightarrow I^{\otimes i} \otimes E$ are null by [51, Proposition 2.5(2)], so the colimit vanishes as desired.

(2) We first show that each $\tau_{\leq n} X_{n}$ is strongly $R$-nilpotent and, more generally, that if $Y$ is $E$-nilpotent, then each $\pi_{i}(Y)$ is strongly $R$-nilpotent. By Lemma 2.6(1) we may assume $Y$ is a (free) $E$-module; in this case, each $\pi_{i}(Y)$ is a $\pi_{0}(E)$-module. Suppose $Y \in \mathcal{C}$ is strongly $\pi_{0}(E)$-nilpotent. Then $Y$ is $E$-nilpotent since any $\pi_{0}(E)$-module is an $E$-module. Finally, we have

$$
\operatorname{colim}_{n}\left[\tau_{\leq n} X_{n}, Y\right] \simeq \underset{n}{\operatorname{colim}}\left[X_{n}, Y\right] \simeq[X, Y]
$$

Here the first equivalence holds since $Y$ is bounded above and the second because $Y$ is $E$-nilpotent.

Next we prove that the $E$-nilpotent completion only depends on $\pi_{0}(E)$.

Proof of Theorem 2.1. For $E \in \mathrm{CA} \lg \left(\mathcal{C}_{\geq 0}\right)$ and $X \in \mathcal{C}$, denote by $R_{n}(E, X)$ the $n$th partial totalisation of $X \otimes E^{\otimes \bullet}$, so that $X \rightarrow R_{\bullet}(E, X)$ is a tower with limit $X \rightarrow X_{E}^{\wedge}$. By left completeness and cofinality 
we have

$$
X_{E}^{\wedge} \simeq \lim _{m, n} \tau_{\leq m} R_{n}(X, E) \simeq \lim _{n} \tau_{\leq n} R_{n}(X, E)
$$

By Lemma 2.7, the right-hand side is the limit of a strongly $\pi_{0}(E)$-nilpotent resolution, which by Proposition 3 only depends on $X$ and $\pi_{0}(E)$.

Remark 2.8. The proof also verifies that any strongly $\pi_{0}(E)$-nilpotent resolution of $X$ has limit $X_{\pi_{0} E}^{\wedge}$.

We now turn to the study of $x$-completions.

Lemma 2.9. Let $L_{1}, \ldots, L_{n} \in \mathcal{C}$ be strongly dualisable and $x_{i}: L_{i} \rightarrow 1$. Let $Y \in \mathcal{C}$ and suppose that, for every $i$, the map

$$
Y \otimes L_{i} \stackrel{x_{i}}{\longrightarrow} Y
$$

is null. Then there is an equivalence

$$
\underset{m_{1}, \ldots, m_{n}}{\operatorname{colim}} \operatorname{map}\left(X /\left(x_{1}^{m_{1}}, \ldots, x_{n}^{m_{n}}\right), Y\right) \simeq \operatorname{map}(X, Y) .
$$

Proof. As a first observation, note that the maps $Y \otimes L_{i} \stackrel{x_{i}}{\longrightarrow} Y$ and $Y \stackrel{D x_{i}}{\longrightarrow} Y \otimes D L_{i}$ correspond under the equivalence $\operatorname{Map}\left(Y \otimes L_{i}, Y\right) \simeq \operatorname{Map}\left(Y, Y \otimes D\left(L_{i}\right)\right)$. It follows that $D x_{i}$ is null.

First consider the case $n=1$. By definition we have $\operatorname{fib}\left(X \rightarrow X / x^{m}\right) \simeq X \otimes L^{\otimes m}$. Hence, it suffices to prove $\operatorname{colim}_{m} \operatorname{map}\left(X \otimes L^{\otimes m}, Y\right)=0$. This term can be identified with colim $m \operatorname{map}\left(X,(D L)^{\otimes m} \otimes Y\right)$, and the transition maps in this system are null by our first observation. In the general case, we note the equivalence

$$
X /\left(x_{1}^{m_{1}}, \ldots, x_{n}^{m_{n}}\right) \simeq\left(X / x_{1}^{m_{1}}\right) /\left(x_{2}^{m_{2}}, \ldots, x_{n}^{m_{n}}\right)
$$

Hence, we get

$$
\begin{aligned}
\operatorname{colim}_{m_{1}, \ldots, m_{n}} \operatorname{map}\left(X /\left(x_{1}^{m_{1}}, \ldots, x_{n}^{m_{n}}\right), Y\right) & \simeq \operatorname{colim}_{m_{1}} \operatorname{colim} \operatorname{map}\left(\left(X / x_{1}^{m_{1}}\right) /\left(x_{2}^{m_{2}}, \ldots, m_{n} x_{n}^{m_{n}}\right), Y\right) \\
& \simeq \operatorname{colim}_{m_{1}} \operatorname{map}\left(X / x_{1}^{m_{1}}, Y\right) \\
& \simeq \operatorname{map}(X, Y) .
\end{aligned}
$$

The first equivalence holds since colimits commute and the other two hold by induction.

Lemma 2.10. Suppose $L \in \mathcal{C}_{\geq 0}$ is strongly dualisable with strong dual $D L \in \mathcal{C}_{\geq 0}$. Then, for all $X \in \mathcal{C}$, there are equivalences

$$
\pi_{i}(X \otimes L) \simeq \pi_{i}(X) \otimes L \simeq \pi_{i}(X) \otimes \pi_{0}(L) .
$$

Proof. By assumption we have $\mathcal{C}_{\geq 0} \otimes L \subset \mathcal{C}_{\geq 0}$. The same holds for $D L$, which implies $\mathcal{C}_{\leq 0} \otimes L \subset \mathcal{C}_{\leq 0}$. In other words, $\otimes L: \mathcal{C} \rightarrow \mathcal{C}$ is $t$-exact and hence $\pi_{i}(X \otimes L) \simeq \pi_{i}(X) \otimes L$. Being in the heart $\mathcal{C}^{\ominus}$, the latter tensor product is equivalent to $\pi_{i}(X) \otimes^{\odot} \pi_{0}(L)$.

Let us quickly verify that $\pi_{0}\left(\mathbf{1} /\left(x_{1}, \ldots, x_{n}\right)\right)$ is indeed an idempotent algebra in $\mathcal{C}^{\diamond}$.

Lemma 2.11. Let $L_{1}, \ldots, L_{n} \in \mathcal{C}_{\geq 0}$ and $x_{i}: L_{i} \rightarrow$ 1. Then $R=\pi_{0}\left(\mathbf{1} /\left(x_{1}, \ldots, x_{n}\right)\right)$ defines an idempotent object of $\mathrm{CAlg}\left(\mathcal{C}^{\diamond}\right)$ and the multiplication maps $\pi_{0}\left(L_{i}\right) \otimes \otimes^{\ominus} \stackrel{x_{i}}{\longrightarrow} R$ are null. 
Proof. Recall that idempotent commutative algebras in $\mathcal{C}^{\diamond}$ are the same as maps $\pi_{0}(\mathbf{1}) \rightarrow A \in \mathcal{C}^{\diamond}$ such that the induced map $A \rightarrow A \otimes^{\ominus} A$ is an isomorphism [49, Proposition 4.8.2.9]. Note that

$$
\pi_{0}\left(1 /\left(x_{1}, \ldots, x_{n}\right)\right) \simeq \pi_{0}\left(\pi_{0}\left(1 /\left(x_{1}, \ldots, x_{n-1}\right)\right) / x_{n}\right) .
$$

More generally, let us prove that if $\pi_{0}(\mathbf{1}) \rightarrow A \in \mathcal{C}^{\diamond}$ is an idempotent algebra and $L \in \mathcal{C}_{\geq 0}, x$ : $L \rightarrow \mathbf{1}$, then $\pi_{0}(A / x)$ is also an idempotent algebra on which multiplication by $x$ is null. Consider the commutative diagram of cofibre sequences

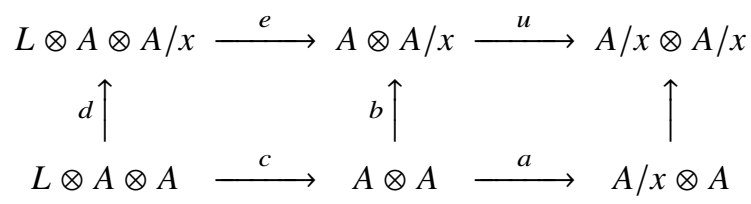

Here $c$ and $e$ 'multiply $L$ into the left factor $A$ ' and all of the other maps are the canonical projections. Since $A$ is idempotent, $\pi_{0}(A \otimes A) \simeq A$ and $\pi_{0}(A \otimes A / x) \simeq \pi_{0}(A / x) \simeq \pi_{0}(A / x \otimes A)$. Under these identifications we have $\pi_{0}(a)=\pi_{0}(b)$ and so $\pi_{0}(e d)=\pi_{0}(b c)=\pi_{0}(a c)=0$. Since $\pi_{0}(d)$ is an epi we deduce $\pi_{0}(e)=0$, and hence $\pi_{0}(u)$ is an isomorphism. This concludes the proof since, under our identifications, $\pi_{0}(e)$ is multiplication by $x$ on $\pi_{0}(A / x)$ and $\pi_{0}(u)$ is $\pi_{0}(A / x) \rightarrow \pi_{0}(A / x) \otimes^{\ominus} \pi_{0}(A / x)$.

We can now identify $x$-completions as $E$-nilpotent completions for an appropriate $E$.

Proof of Theorem 2.2. Lemma 2.11 shows $R_{n}=\pi_{0}\left(\mathbf{1} /\left(x_{1}, \ldots, x_{n}\right)\right)$ is idempotent.

Step 1: The map $R_{n} \otimes L_{i} \stackrel{x_{i}}{\longrightarrow} R_{n}$ is null. Indeed, by Lemma 2.10, we have $R_{n} \otimes L_{i} \simeq R_{n} \otimes{ }^{\ominus} \pi_{0}\left(L_{i}\right)$, and so this follows from Lemma 2.11.

Step 2: We show the homotopy objects of $X /\left(x_{1}^{e_{1}}, \ldots, x_{n}^{e_{n}}\right)$ are strongly $R_{n}$-nilpotent for all $e_{i} \geq 1$. By an induction argument, using the octahedral axiom, $X / x^{m}$ is a finite extension of copies of $X / x$. Hence, each $X /\left(x_{1}^{e_{1}}, \ldots, x_{n}^{e_{n}}\right)$ is a finite extension of copies of $X /\left(x_{1}, \ldots, x_{n}\right)$; thus, we may assume $e_{i}=1$. By induction on $n$ and Lemma 2.10, together with Lemma 2.6(1), it suffices to show that if $M \in \mathcal{C}^{\diamond}$ is $R_{i}$-nilpotent, then both the kernel and cokernel of

$$
M \otimes \otimes_{0}\left(L_{i+1}\right) \stackrel{x_{i+1}}{\longrightarrow} M
$$

are $R_{i+1}$-nilpotent. The proof given in [50, Lemma 7.2.10] goes through unchanged in our setting.

Step 3: We show that

$$
\left\{\tau_{\leq m} X /\left(x_{1}^{e_{1}}, \ldots, x_{n}^{e_{n}}\right)\right\}_{e_{1}, \ldots, e_{n} ; m}
$$

is a strongly $R_{n}$-nilpotent resolution of $X$. Since we assume $X$ is connected, step 2 shows

$$
\tau_{\leq m} X /\left(x_{1}^{e_{1}}, \ldots, x_{n}^{e_{n}}\right)
$$

is bounded with strongly $R_{n}$-nilpotent homotopy objects. Owing to Lemma 2.6(2), it is in fact strongly $R_{n}$-nilpotent. We thus need to show that if $Y$ is strongly $R_{n}$-nilpotent, then

$$
\operatorname{colim} \operatorname{map}\left(\tau_{\leq m} X /\left(x_{1}^{e_{1}}, \ldots, x_{n}^{e_{n}}\right), Y\right) \simeq \operatorname{map}(X, Y) .
$$

Since $Y$ is bounded above, we may remove $\tau_{\leq m}$ in the above expression without changing the colimit. We may assume that $Y$ is an $R_{n}$-module in $\mathcal{C}^{\ominus}$. By step 1 the map $L_{i} \otimes Y \rightarrow Y$ is null, and so the claim follows from Lemma 2.9.

Conclusion of proof: By left completeness we have

$$
X_{x_{1}, \ldots, x_{n}}^{\wedge} \simeq \lim _{e_{1}, \ldots, e_{n} ; m} \tau_{\leq m} X /\left(x_{1}^{e_{1}}, \ldots, x_{n}^{e_{n}}\right) .
$$


According to step 3, this is the limit of a strongly $R_{n}$-nilpotent resolution of $X$, which coincides with $X_{R_{n}}^{\wedge}$ by Remark 2.8 .

\section{Rigidity for stable motivic homotopy of henselian local schemes}

In this section we prove results to the effect that if $X$ is a suitable henselian local scheme with closed point $x$ and $E$ is an appropriate motivic spectrum, then $E(X) \simeq E(x)$. Such results are known as 'rigidity'. Many instances have been proved before, mainly if $X$ is essentially smooth over a field; see, for example, $[35,1]$. Our main novelty is that we replace the base by a Dedekind domain, at the cost of imposing much stronger assumptions on $E$.

Given a presentably symmetric monoidal stable $\infty$-category $\mathcal{C}$ and a morphism $a: L \rightarrow \mathbf{1}$ with $L$ strongly dualisable, we denote by $\mathcal{C}_{a}^{\wedge}$ the $a$-completion; that is, the localisation at maps which become an equivalence after $\otimes \operatorname{cof}(a)$. We refer to [7, §2.1], [10, §2.5] for more details; in particular, the $a$-completion of $X$ is given by the object $X_{a}^{\wedge}$ from the previous section.

Given a family of objects $\mathcal{G} \subset \mathcal{C}$ (which for us will always be bigraded spheres $\Sigma^{* *} \mathbf{1}$ ), we write $\mathcal{C}^{\text {cell }}$ for the localising subcategory generated by $\mathcal{G}$. Noting that $\mathcal{C}_{a}^{\wedge}$ is equivalent to the localising tensor ideal generated by $\operatorname{cof}(a)$, by, for example, [7, Example 2.3], we see that if $L \in \mathcal{G}$, then these two operations commute, and so we shall write

$$
\mathcal{C}_{a}^{\wedge \text { cell }}:=\left(\mathcal{C}_{a}^{\wedge}\right)^{\text {cell }} \simeq\left(\mathcal{C}^{\text {cell }}\right)_{a}^{\wedge}
$$

Recall the element $h:=1+\langle-1\rangle \in \pi_{0,0}(\mathbf{1})$, where $-\langle-1\rangle$ is the switch map on $\mathbb{G}_{m} \wedge \mathbb{G}_{m}$, and the element $\rho:=[-1] \in \pi_{-1,-1}(\mathbf{1})$ corresponding to $-1 \in \mathcal{O}^{\times}$.

Proposition 4. Suppose $X$ is a henselian local scheme and essentially smooth over a Dedekind scheme. Write $i: x \rightarrow X$ for the inclusion of the closed point and let $n \in \mathbb{Z}$.

1. If $1 / n \in X$, then $i^{*}: \mathcal{S H}(X)_{n}^{\wedge c e l l} \rightarrow \mathcal{S H}(x)_{n}^{\wedge c e l l}$ is an equivalence.

2. If $1 / 2 n \in X$, then $i^{*}: \mathcal{S H}(X)_{n h}^{\wedge c e l l} \rightarrow \mathcal{S H}(x)_{n h}^{\wedge c e l l}$ is an equivalence.

3. $i^{*}: \mathcal{S H}(X)\left[\rho^{-1}\right]^{\text {cell }} \rightarrow \mathcal{S H}(x)\left[\rho^{-1}\right]^{\text {cell }}$ is an equivalence.

Many proofs in the sequel will follow the pattern of this one. We spell out many details here, which are suppressed in the following proofs.

Proof. If $S$ is a quasi-compact quasi-separated scheme - for example, affine - the category $\mathcal{S H}(S)$ is compactly generated by suspension spectra of finitely presented smooth $S$-schemes [37, Proposition C.12]. Thus, $\mathcal{S H}(S)^{\text {cell }}$ is compactly generated by the spheres, and for every $a \in \pi_{* *}\left(\mathbf{1}_{S}\right)$, the category $\mathcal{S H}(S)_{a}^{\wedge \text { cell }}$ is compactly generated by $\Sigma^{* *} \mathbf{1} / a$. Now let $f: S^{\prime} \rightarrow S$ be a morphism, where $S^{\prime}$ is also quasi-compact quasi-separated. We use $f^{*}$ to transport elements of $\pi_{* *}\left(\mathbf{1}_{S}\right)$ to $\pi_{* *}\left(\mathbf{1}_{S^{\prime}}\right)$, and when no confusion can arise, we denote them by the same letter. Thus, for example, we set

$$
\mathcal{S H}\left(S^{\prime}\right)_{a}^{\wedge}:=\mathcal{S H}\left(S^{\prime}\right)_{f^{*} a}^{\wedge}
$$

The functor $f^{*}: \mathcal{S H}(S)_{a}^{\wedge \text { cell }} \rightarrow \mathcal{S H}\left(S^{\prime}\right)_{a}^{\wedge \text { cell }}$ preserves colimits and the compact generator. Therefore, it admits a right adjoint $f_{*}$ preserving colimits. This implies that $f^{*}$ is fully faithful if and only if the map $\mathbf{1} \rightarrow f_{*} f^{*} \mathbf{1} \in \mathcal{S H}(S)_{a}^{\wedge \text { cell }}$ is an equivalence; see, for example, [4, Lemma 22]; in this case, the functor is an equivalence since its essential image will be a localising subcategory containing the generator.

We can simplify this condition further. By a-completeness and Lemma 3.1, it follows that $\mathbf{1} \rightarrow f_{*} f^{*} \mathbf{1}$ is an equivalence if and only if $\mathbf{1} / a \rightarrow f_{*} f^{*}(\mathbf{1} / a)$ is an equivalence; that is, if and only if

$$
\pi_{* *}\left(\mathbf{1}_{S} / a\right) \simeq \pi_{* *}\left(\mathbf{1}_{S^{\prime}} / a\right) .
$$


If $b \in \pi_{* *}(\mathbf{1})$, then in our compactly generated situations the $b$-periodisation $\mathcal{E}\left[b^{-1}\right]$ is given by the colimit

$$
\mathcal{E}\left[b^{-1}\right]=\operatorname{colim}\left(\mathcal{E} \stackrel{b}{\rightarrow} \Sigma^{* *} \mathcal{E} \stackrel{b}{\rightarrow} \ldots\right)
$$

Since $f_{*}$ preserves colimits, it commutes with $b$-periodisation by Lemma 3.1. We shall make use of the fact that a map is an equivalence if and only if it is an equivalence after $b$-periodisation and $b$-completion; see, for example, [10, Lemma 2.16]. Thus, to prove fully faithfulness it would also be sufficient, as well as necessary, to prove

$$
\pi_{* *}\left(\mathbf{1}_{S} /(a, b)\right) \simeq \pi_{* *}\left(\mathbf{1}_{S^{\prime}} /(a, b)\right) \quad \text { and } \quad \pi_{* *}\left(\mathbf{1}_{S}\left[b^{-1}\right] / a\right) \simeq \pi_{* *}\left(\mathbf{1}_{S^{\prime}}\left[b^{-1}\right] / a\right) .
$$

We will use many different variants of these observations in the sequel.

(0) We claim the functor

$$
\mathcal{S H}(X)\left[\eta^{-1}\right] \rightarrow \mathcal{S H}(x)\left[\eta^{-1}\right]
$$

is an equivalence provided $1 / 2 \in X$ and that

$$
\mathcal{S H}(X)\left[\eta^{-1}, 1 / 2\right] \rightarrow \mathcal{S H}(x)\left[\eta^{-1}, 1 / 2\right]
$$

is an equivalence without any assumptions on $X$. For the first claim, by the above remarks it suffices to prove that $\pi_{* *}\left(\mathbf{1}\left[\eta^{-1}\right]\right)$ satisfies the required rigidity, which via [6, Proposition 5.2] reduces to the same statement for the Witt ring $W(-)$. This is true by [41, Lemma 4.1]. Since $\mathcal{S H}(S)\left[\eta^{-1}, 1 / 2\right] \simeq$ $\mathcal{S H}(S)\left[\rho^{-1}, 1 / 2\right]$ (see Lemma 3.2), the second claim reduces to (3).

(1) It suffices to establish an isomorphism on $\eta$-periodisation and $\eta$-completion. We first treat the $\eta$-complete case; that is, we need to show that $\mathbf{1} \rightarrow i_{*} i^{*} \mathbf{1} \in \mathcal{S} \mathcal{H}_{n, \eta}^{\wedge \text { cell }}$ is an equivalence. By Theorem 2.3(2) with $\ell=n$, we have

$$
E_{n, \eta}^{\wedge} \simeq \lim _{\Delta} E \wedge H \mathbb{Z} / n^{\wedge \bullet+1}
$$

for any bounded below $E$ in $\mathcal{S H}(S)$. The cellularisation functor $\mathcal{S H}(S) \rightarrow \mathcal{S H}(S)^{\text {cell }}$ preserves limits and hence $(n, \eta)$-completions. Moreover, $\mathrm{HZ} / n \in \mathcal{S H}(S)^{\text {cell }}$ if $1 / n \in S$ by [69, Corollary 10.4]. Hence, the above formula for $E_{n, \eta}^{\wedge}$ also makes sense, and is true, in $\mathcal{S H}(S)^{\text {cell }}$. Thus, we need to show the map $\mathrm{HZ} / 2^{\wedge t} \rightarrow i_{*}\left(\mathrm{HZ} / 2^{\wedge t}\right) \in \mathcal{S H}(X)^{\text {cell }}$ is an equivalence, for $t \geq 1$. Lemma 3.1 implies that $i_{*}\left(E \wedge i^{*} F\right) \simeq i_{*}(E) \wedge F$, for any $E \in \mathcal{S H}(x), F \in \mathcal{S H}(X)^{\text {cell }}$. In this way, we reduce to $t=1$; that is, it suffices to show

$$
\pi_{* *}\left(\mathrm{H} \mathbb{Z} / n_{X}\right) \simeq \pi_{* *}\left(\mathrm{H} \mathbb{Z} / n_{x}\right) .
$$

Owing to [69, Theorem 3.9], $\pi_{* *}\left(\mathrm{HZ} / n_{S}\right)$ is given by the Zariski cohomology of $S$ with coefficients in a truncation of the étale cohomology of $\mu_{n}^{\otimes-}$. When $S=X$ or $S=x$, the scheme $S$ is Zariski local, so $\pi_{* *}\left(\mathrm{HZ} / n_{S}\right)$ is simply given by certain étale cohomology groups of $S$ with coefficients in $\mu_{n}^{\otimes-}$. The rigidity result follows now from [28, Theorem 1].

Next we treat the $\eta$-periodic case. If $n$ is even, then $1 / 2 \in X$ and so the result follows from (0). If $n$ is odd, then $n$-complete objects are 2-periodic and the result also follows from (0).

(2) Again it suffices to prove that we have an isomorphism after $\eta$-completion and $\eta$-periodisation; (0) handles the $\eta$-periodic case. For the $\eta$-complete case, we use that $\pi_{0}(\mathbf{1} /(n h, \eta)) \simeq \pi_{0}(1 /(2 n, \eta))$ (see Lemma 3.2), whence $\mathbf{1}_{n h, \eta}^{\wedge} \simeq \mathbf{1}_{2 n, \eta}^{\wedge}$ by Theorem 2.2; this reduces to (1).

(3) By [5, Theorem 35] we have $\mathcal{S H}(S)\left[\rho^{-1}\right] \simeq \mathcal{S H}\left(S_{\text {rét }}\right)$, where the right-hand side denotes hypersheaves on the small real étale site of $S$. In this situation we have a natural $t$-structure; see, for example, [7, §2.2], such that the map $\mathbf{1}_{\text {rét }} \rightarrow \mathrm{H}_{\text {rét }} \mathbb{Z}$ is a morphism of connective ring spectra inducing 
an isomorphism on $\pi_{0}$, where by $\mathrm{H}_{r e ́ t} \mathbb{Z}$ we mean the constant sheaf of spectra. Hence, applying Theorem 2.1 in this situation, and repeating the above discussion using that $\mathrm{H}_{\text {rét }} \mathbb{Z}$ is cellular and stable under base change, essentially by definition, we find that in order to prove $\mathbf{1} \rightarrow i_{*} i^{*} \mathbf{1} \in \mathcal{S H}(S)\left[\rho^{-1}\right]^{\text {cell }}$ is an equivalence, it suffices to prove $\mathrm{H}_{\text {rét }} \mathbb{Z} \rightarrow i_{*} \mathrm{H}_{\text {rét }} \mathbb{Z}$ is an equivalence. In other words, we need to show

$$
H_{\text {rét }}^{*}(X, \mathbb{Z}) \simeq H_{\text {rét }}^{*}(x, \mathbb{Z}) .
$$

Since the real étale and Zariski cohomological dimension coincide [65, Theorem 7.6], we are reduced to $H_{r e ́ t}^{0}$, which follows from [2, Propositions II.2.2, II.2.4].

Lemma 3.1. Let $F: \mathcal{C} \rightarrow \mathcal{D}$ be a symmetric monoidal functor between symmetric monoidal categories admitting a right adjoint $G$, and let $x: A \rightarrow \mathbf{1}$ be a morphism in $\mathcal{C}$ with $A$ strongly dualisable. Then for $X \in \mathcal{D}$, there is a natural equivalence $G(X \otimes F A) \simeq G(X) \otimes A$, and under this equivalence the map

$$
G(X \otimes F A) \stackrel{G(\operatorname{id} \otimes F x)}{\longrightarrow} G(X)
$$

corresponds to

$$
G X \otimes A \stackrel{x}{\rightarrow} G X
$$

Suppose that $\mathcal{C}, \mathcal{D}$ are presentably symmetric monoidal stable $\infty$-categories and $G$ preserves colimits. Write $\mathcal{C}^{\prime}$ for the localising subcategory of $\mathcal{C}$ generated by strongly dualisable objects. Then the above result also holds for any $A \in \mathcal{C}^{\prime}$.

Proof. Since $F$ is symmetric monoidal, $G$ is lax symmetric monoidal, and there is a canonical map $G X \otimes G F A \rightarrow G(X \otimes F A)$. Composing with the unit $A \rightarrow G F A$, we obtain a natural map $G X \otimes A \rightarrow$ $G(X \otimes F A)$, which is an equivalence by the Yoneda lemma. Since this equivalence is natural in $A$ as well, the claim about $x$ also follows.

For the second statement, the subcategory comprising $A \in \mathcal{C}$ for which the natural transformation $G X \otimes A \rightarrow G(X \otimes F A)$ is an equivalence for all $X \in \mathcal{D}$ is localising since $G$ preserves colimits and it contains all strongly dualisable objects by the first part and hence all of $\mathcal{C}^{\prime}$.

Lemma 3.2. In $\pi_{*, *}(\mathbf{1})$ we have the relations

$$
\eta h=0, h=2+\eta \rho, h \rho^{2}=0 .
$$

It follows that

$$
\mathcal{S H}(S)[1 / 2,1 / \eta] \simeq \mathcal{S H}(S)[1 / 2,1 / \rho]
$$

Proof. By [19, Theorem 1.2], all of the Milnor-Witt relations hold in $\pi_{*, *}(\mathbf{1})$, including $\eta h=0$. Our definition of $h$ agrees with Druzhinin's by [19, Lemma 3.10]. We now compute

$$
h \rho^{2}=(2+\eta[-1])[-1][-1]=2[-1][-1]+([1]-[-1]-[-1])[-1]=0
$$

using the logarithm relation $[a b]=[a]+[b]+\eta[a][b]$ as well as $[1]=0$, which holds by definition.

For the last part, note that inverting either $\eta$ or $\rho$ kills $h$ (by the first or third relation) and hence makes $\eta$ and $\rho$ inverses of each other up to a factor of $-1 / 2$ (by the second relation).

Example 3.3. Suppose that $1 / 2 \in X$, where $X$ is henselian local and essentially smooth over a Dedekind scheme. Applying Proposition 4(2) with $n=1$, we learn that $\mathcal{S H}(X)_{h}^{\wedge \text { cell }} \rightarrow \mathcal{S H}(x)_{h}^{\wedge \text { cell }}$ is an equivalence. By Lemma 3.2, both the $\eta$-periodic and $\rho$-periodic objects are $h$-torsion. We conclude 
$\mathcal{S H}(X)_{h}^{\wedge}\left[\eta^{-1}\right] \simeq \mathcal{S H}(X)\left[\eta^{-1}\right]$ and similarly for $\rho$. Thus, there is an equivalence

$$
\mathcal{S H}(X)\left[\eta^{-1}\right]^{\text {cell }} \simeq \mathcal{S H}(x)\left[\eta^{-1}\right]^{\text {cell }} .
$$

A similar equivalence holds for $\rho$. With reference to Proposition 4, this shows (2) implies (3).

Example 3.4. We have $\left(E_{a b}^{\wedge}\right)_{a}^{\wedge} \simeq E_{a}^{\wedge}$ since $a b$-periodic objects are $a$-periodic. Hence, in Proposition 4, (2) implies (1).

\section{Topological models for stable motivic homotopy of regular number rings}

We shall exhibit pullback squares describing $\mathcal{S H}\left(\mathcal{O}_{F}[1 / \ell]\right)_{\ell}^{\wedge c e l l ~}$ for suitable number fields $F$ and prime numbers $\ell$ in terms of $\mathcal{S H}(k)_{\ell}^{\wedge \text { cell }}$ for fields of the form $k=\mathbb{C}, \mathbb{R}, \mathbb{F}_{q}$. To facilitate comparison with the work of Dwyer-Friedlander [24], we formally dualise our terminology and exhibit pushout squares in the opposite category.

\subsection{Setup}

Let $\ell$ be a prime (or, more generally, any integer, but we do not need or use this extra generality). We shall use the notation $\ell^{\prime}=\ell$ if $\ell$ is odd and $\ell^{\prime}=\ell h$ if $\ell=2$.

\section{Definition 5.}

1. We write

$$
\mathcal{C M}_{S} \subset\left(\operatorname{CAlg}\left(\mathcal{P r}^{\mathrm{L}}\right)^{\mathrm{op}}\right)_{/ \mathcal{S H}(S)^{\text {cell }}}
$$

for the full subcategory comprising functors $F: \mathcal{S H}(S)^{\text {cell }} \rightarrow \mathcal{C}$, where $\mathcal{C}$ is generated under colimits by $F\left(\mathcal{S H}(S)^{\text {cell }}\right)$ (or, equivalently, by $F\left(S^{p, q}\right)$ for $p, q \in \mathbb{Z}$ ).

2. We denote by $M_{\ell^{\prime}}$ the functor

$$
\operatorname{Sch}_{\mathbb{Z}[1 / \ell]} \rightarrow \mathcal{C M}_{\mathbb{Z}[1 / \ell]}, \quad X \mapsto \mathcal{S H}(X)_{\ell^{\prime}}^{\wedge \text { cell }}, \quad(f: X \rightarrow Y) \mapsto\left(f^{*}: \mathcal{S H}(Y)_{\ell^{\prime}}^{\wedge \text { cell }} \rightarrow \mathcal{S H}(X)_{\ell^{\prime}}^{\wedge \text { cell }}\right)^{\mathrm{op}} .
$$

We also put $\mathcal{C M}=\mathcal{C M}_{\mathbb{Z}}$ and, by abuse of notation, $M(X):=M_{0}(X)=\mathcal{S H}(X)^{\text {cell }} \in \mathcal{C M}$. Note that $\mathcal{C M}_{S}=\mathcal{C M}_{/ M_{0}(S)}$ and $M_{\ell^{\prime}}(X)=M(X)_{\ell^{\prime}}^{\wedge}$. Next, we clarify the meaning of colimits in $\mathcal{C M}_{S}$.

Lemma 4.1. Let $F: I \rightarrow \mathcal{C M} \mathcal{M}_{S}$ be a diagram and write $F^{\prime}: I^{\mathrm{op}} \rightarrow \mathcal{C a t}_{\infty}$ for the underlying diagram of categories. Then $\lim _{I^{\text {op }}} F^{\prime} \in \mathcal{C} \mathrm{at}_{\infty}$ is presentably symmetric monoidal and admits a natural functor from $\mathcal{S H}(S)^{\text {cell }}$. Let $\mathcal{C}$ denote its subcategory generated under colimits by the image of $\mathcal{S H}(S)^{\text {cell }}$. Then there is an equivalence $\operatorname{colim}_{I} F \simeq \mathcal{C}$.

Proof. The forgetful functor

$$
\mathrm{CA} \lg \left(\mathcal{P r}^{\mathrm{L}}\right)_{\mathcal{S H}(S)^{\mathrm{cell}} /} \rightarrow \mathcal{C a t}_{\infty}
$$

preserves limits [48, Propositions 5.5.3.13, 1.2.13.8], [49, Corollary 3.2.2.5], and hence the limit admits a canonical functor from $\mathcal{S H}(S)^{\text {cell }}$. For $\mathcal{D} \in \mathcal{C M}$ we have

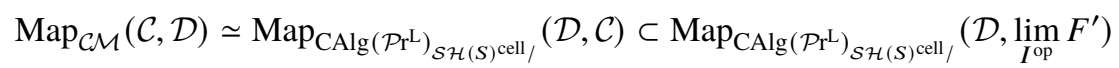

$$
\begin{aligned}
& \simeq \lim _{I^{\mathrm{op}}} \operatorname{Map}_{\mathrm{CAlg}\left(\mathcal{P r}^{\mathrm{L}}\right)_{\mathcal{S H}(S)^{\mathrm{cell}} /}}(F(-), \mathcal{D}) \simeq \lim _{I^{\mathrm{op}}} \operatorname{Map}_{\mathcal{C M}}(F(-), \mathcal{D}) .
\end{aligned}
$$

It remains to show the inclusion is an equivalence; that is, every map $\mathcal{D} \rightarrow \lim _{I^{\text {op }}} F^{\prime}$ in $\mathrm{CAlg}\left(\mathcal{P r}^{\mathrm{L}}\right)_{\mathcal{S H}(S)^{\text {cell }}}$ factors through $\mathcal{C}$. This holds for the generators, by assumption, so we are done. 
Next we reformulate and slightly extend our rigidity results from Section 3.

Lemma 4.2. Let $\bar{x}$ be the spectrum of a separably closed field, $X \in \mathrm{Sch}_{\mathbb{Z}[1 / \ell]}$ an essentially smooth over a Dedekind domain, $\bar{x} \rightarrow X$ a map and $y \in X$ a specialisation of the image of $x$. In $\mathcal{C M}_{\mathbb{Z}[1 / \ell]}$ there is a commutative diagram

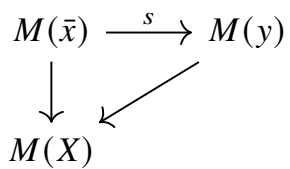

Here the unlabelled maps are the canonical ones. In fact, there is a family of such commutative diagrams, parametrised by the (nonempty) set $X_{y}^{h} \times_{X} \bar{x}$.

Proof. Let $X^{\prime}$ be the henselisation of $X$ along $y$. By [71, Tags 03HV, 07QM(1)], the map $X^{\prime} \rightarrow X$ hits the image of $\bar{x}$, and hence there exists a lift $s^{\prime}$ in the commutative diagram

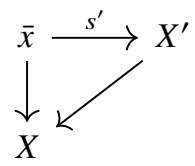

Applying $M$ and using that $M(y) \rightarrow M\left(X^{\prime}\right)$ is an equivalence by Proposition 4, the result follows.

Corollary 6. The following hold under the assumptions in Lemma 4.2.

1. If $y \in X$ is separably closed, then $s$ is an equivalence.

2. If $\bar{x}, \bar{y} \in \mathrm{Sch}_{\mathbb{Z}[1 / \ell]}$ are separably closed fields there is a (nonunique) equivalence $M(\bar{x}) \simeq M(\bar{y})$.

Proof. (1) We have constructed a symmetric monoidal cocontinuous functor $F: \mathcal{S H}(\bar{y})_{\ell^{\prime}}^{\wedge \text { cell }} \rightarrow$ $\mathcal{S H}(\bar{x})_{\ell^{\prime}}^{\wedge \text { cell }}$ under $\mathcal{S H}(\mathbb{Z}[1 / \ell])^{\text {cell }}$. Denote its right adjoint by $G$. Arguing as in the proof of Proposition 4, it suffices to show $\mathcal{E} \rightarrow G F \mathcal{E}$ is an equivalence. That is, $\mathcal{E} \rightarrow G F \mathcal{E}$ induces an isomorphism on $\pi_{* *}$ for $\mathcal{E}=\mathrm{HF}_{\ell}, \mathcal{E}=\mathrm{H}_{\text {rét }} \mathbb{Z}$ and, if $\ell$ is even, $\mathcal{E}=\mathbf{1}\left[\eta^{-1}\right]$. For any separably closed field of characteristic $\neq \ell$ we have $\pi_{* *}\left(\mathrm{HF}_{\ell}\right) \simeq \mathbb{F}_{\ell}[\tau]$ (see, for example, [12, Corollary C.2(2)], [40, Theorem 18.2.7]), $W=\mathbb{Z} / 2$ and $\mathrm{H}_{\text {rét }} \mathbb{Z}=0$ (the real spectrum being empty). Moreover, all of the maps are algebra maps over the corresponding algebra for $\mathbb{Z}[1 / \ell]$. Thus, the map for $\pi_{* *} \mathrm{H}_{r e ́ t} \mathbb{Z}$ is trivially an isomorphism, and the one for $\pi_{* *} \mathbf{1}\left[\eta^{-1}\right]$ is an isomorphism because as an algebra it is determined by $W$ according to [6, Proposition 5.2]. The isomorphism for $\mathrm{HF}_{\ell}$ will hold if and only if $F(\tau)=\tau$, which holds provided $F\left(\tau^{n}\right)=\tau^{n}$ for some $n \geq 1$. But, for $n \gg 0, \tau^{n}$ exists over $\mathbb{Z}[1 / \ell]$ (if $\ell=2$, this holds with $n=1$ and for $\ell$ odd; see, e.g., [9, $\$ 4.5(2)])$.

(2) Let $x, y \in \operatorname{Spec}(\mathbb{Z}[1 / \ell])$ be the images of $\bar{x}, \bar{y}$. We may assume $y$ is a specialisation of $x$. Let $X$ be the strict henselisation of $\operatorname{Spec}(\mathbb{Z}[1 / \ell])$ along $y$, with closed point $y^{\prime}$. By (1) applied with $X=X^{\prime}$ we have $M\left(y^{\prime}\right) \simeq M(\bar{x})$, and by applying it with $(X, \bar{x}, y)=\left(\left\{y^{\prime}\right\}, \bar{y}, y^{\prime}\right)$ we get $M\left(y^{\prime}\right) \simeq M(\bar{y})$.

Remark 4.3. This common category $M(\bar{x}) \simeq M(\bar{y})$ is known as $\ell$-complete MU-based (even) synthetic spectra [59].

\subsection{Criterion}

Recall that for $X \in \operatorname{Sch}_{\mathbb{Z}[1 / \ell]}$ the objects $\mathrm{HF}_{\ell}, \mathrm{H}_{\text {rét }} \mathbb{Z} \in \mathcal{S H}(X)$ are cellular and stable under base change. For $\mathrm{HF}_{\ell}$ this is [69, Corollary 10.4, Theorem 8.22]. For $\mathrm{H}_{r e ́ t} \mathbb{Z}$ this follows from the expression $\mathrm{H}_{\text {rét }} \mathbb{Z} \simeq o(\mathrm{HZ})[1 / \rho][5]$, where $o: \mathcal{S H} \rightarrow \mathcal{S H}(X)$ is the unique cocontinuous symmetric monoidal functor. In particular, any morphism between $M(X)$ and $M(Y)$ in $\mathcal{C M}_{\mathbb{Z}[1 / \ell]}$ preserves these objects. 
Proposition 7. Let $X_{0}, X_{1}, X_{2}, X_{3} \in \mathrm{Sch}_{\mathbb{Z}[1 / \ell]}$ be essentially smooth over Dedekind schemes and consider a commutative square

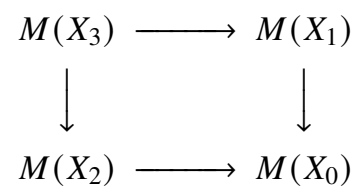

in $\mathcal{C M}_{\mathbb{Z}[1 / \ell]}$. In order for (4.1) to be co-Cartesian, it suffices that the following conditions hold:

1. For each $m$, the square

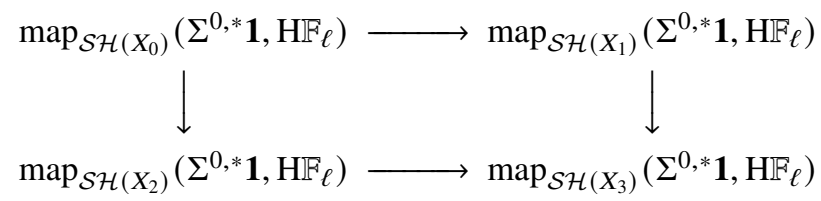

is Cartesian.

2. The square

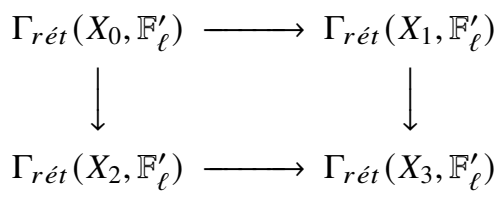

is Cartesian. Here $\mathbb{F}_{\ell}^{\prime}$ equals $\mathbb{F}_{\ell}$ if $\ell=\ell^{\prime}$ and $\mathbb{Z}$ if $\ell^{\prime}=\ell h$ (i.e., when $\ell$ even).

3. If $2 \mid \ell$, then $\operatorname{vcd}_{2}\left(K\left(X_{i}\right)\right)<\infty$.

If $X_{0}$ contains a primitive $\ell$ th root of unity, then condition (1) can be replaced by

(1') For each $m$, the square

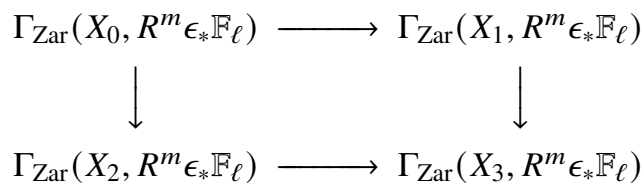

is Cartesian.

Proof. To conclude that the square is co-Cartesian, it suffices, by Lemma 4.1, to prove the functor

$$
\mathcal{S H}\left(X_{0}\right)_{\ell^{\prime}}^{\wedge \text { cell }} \rightarrow \mathcal{S H}\left(X_{1}\right)_{\ell^{\prime}}^{\wedge \text { cell }} \times_{\mathcal{S H}\left(X_{3}\right)_{\ell^{\prime}}^{\wedge \text { cell }}} \mathcal{S H}\left(X_{2}\right)_{\ell^{\prime}}^{\wedge \text { cell }}
$$

is fully faithful. Let us denote by $p_{1 *}: \mathcal{S H}\left(X_{1}\right)_{\ell^{\prime}}^{\wedge \text { cell }} \rightarrow \mathcal{S H}\left(X_{0}\right)_{\ell^{\prime}}^{\wedge \text { cell }}$ the right adjoint of the functor corresponding to $M\left(X_{1}\right) \rightarrow M\left(X_{0}\right)$, and similarly for $p_{2 *}, p_{3 *}$. We need to prove that

$$
\boldsymbol{\pi}_{* *}\left(\mathbf{1}_{\ell^{\prime}}^{\wedge}\right) \simeq \pi_{* *}\left(p_{1 *}\left(\mathbf{1}_{\ell^{\prime}}^{\wedge}\right) \times_{p_{3 *} \mathbf{1}_{\ell^{\prime}}} p_{2 *}\left(\mathbf{1}_{\ell^{\prime}}^{\wedge}\right)\right)
$$

Note that each of the left adjoints preserves the compact generators, which is true for any morphism in $\mathcal{C M}$, and hence $p_{i *}$ preserves colimits and therefore it commutes with periodisation. Moreover, $p_{i *}$ commutes with $\wedge \mathcal{E}$ for every $\mathcal{E} \in \mathcal{S H}\left(X_{0}\right)_{\ell^{\prime}}^{\wedge \text { cell }}$, and with completion at homotopy elements, by Lemma 3.1. We may check the desired equivalence after completing at $\eta$ and after inverting $\eta$, and similarly for other homotopy elements. For the $\eta$-periodic statement, we further invert 2 respectively complete at 2 . In the 2 -complete (still $\eta$-periodic) case, either we have $2 \nmid \ell$ and the statement is vacuous 
or $1 / 2 \in X_{i}$ and using the fundamental fibre sequence [6, Corollary 1.2, Proposition 5.7], it suffices to establish the analogous equivalence for $\mathrm{kw}_{2, \ell^{\prime}}^{\wedge}$. Recall that $\mathrm{kw}_{2, \ell^{\prime}}^{\wedge}$ is in fact cellular [6, Proposition 5.7]. In the 2-periodic (still $\eta$-periodic) case, arguing as in the proof of Proposition 4, it suffices to establish the analogous equivalence for $\mathrm{H}_{r e ́ t} \mathbb{F}_{\ell}^{\prime}$. For the $\eta$-complete statement, arguing as in the proof of Proposition 4, we have $\mathbf{1}_{\eta, \ell^{\prime}}^{\wedge} \simeq \mathbf{1}_{\mathrm{HF} F_{\ell}}^{\wedge}$ and we see that it suffices to establish the analogous equivalence for $\mathrm{HF}_{\ell}$. In summary, we need to prove the commutative square of ordinary spectra

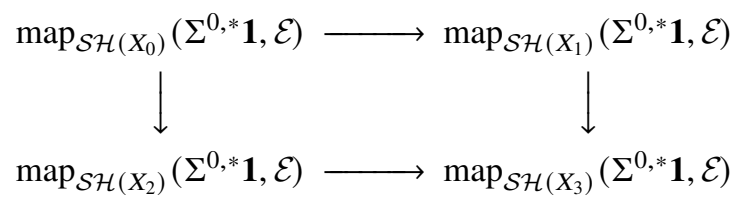

is Cartesian for all $* \in \mathbb{Z}$ and $\mathcal{E}$ one of $\mathrm{kw}_{2, \ell^{\prime}}^{\wedge}, \mathrm{HF}_{\ell}, \mathrm{H}_{\text {rét }} \mathbb{F}_{\ell}^{\prime}$.

Before we start proving this, we need to make another preliminary remark. Suppose that

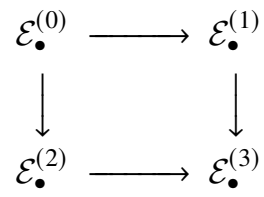

is a commutative diagram of filtered spectra such that $\mathcal{E}_{n}^{(i)}=0$ for $n$ sufficiently small and the induced diagrams of associated graded objects

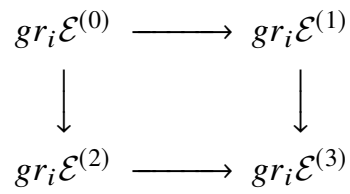

are pullbacks for each $i$. Then the square

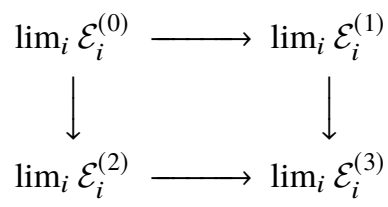

is a pullback; indeed, an induction argument implies

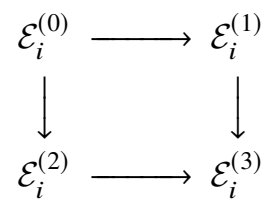

is a pullback for every $i$.

Next we show how the conditions (1)-(3) imply that the squares are Cartesian. The pullback square for $\mathrm{H}_{r e ́ t} \mathbb{F}_{\ell}^{\prime}$ is precisely condition (2), and the one for $\mathrm{HF}_{\ell}$ is precisely condition (1). The condition involving $\mathrm{kw}_{2, \ell^{\prime}}^{\wedge}$ is only nonvacuous if $2 \mid \ell$, whence $1 / 2 \in X_{i}$ and $\mathrm{kw}_{2}^{\wedge} \simeq \mathrm{kw}_{2, \ell^{\prime}}^{\wedge}$. Consider the filtration of kw by powers of $\beta$, pulled back to $X_{i}$. The Postnikov filtration gives rise to the said filtration, and so it is complete, and $\mathrm{HW}$ gives all subquotients [6, Theorem 4.4, Lemma 4.3]. Since kw is connective, the preliminary remark allows us to replace $\mathrm{kw}_{2}^{\wedge}$ by $\mathrm{H} W_{2}^{\wedge}$, which on mapping spectra yields $\Gamma\left(-, \underline{W}_{2}^{\wedge}\right)$, where $\Gamma$ denotes global sections of a Nisnevich sheaf of spectra. On mapping spectra the cellular motivic spectrum $\underline{K}^{W}$ [6, Proposition 5.7, Theorem 4.4] yields compatible filtrations of $\Gamma(-, \underline{W})$ by 
$\Gamma\left(-, \underline{I}^{n}\right)$ (see [6, Definition 2.6]), where $I$ is the fundamental ideal of even dimensional quadratic forms. Condition (3) together with [6, Proposition 2.3] implies $\lim _{n} \Gamma\left(-, \underline{I}^{n} / 2\right) \simeq 0$. Thus, the filtration $\Gamma\left(-,\left(\underline{W} / \underline{I}^{n}\right)_{2}^{\wedge}\right)$ of $\Gamma\left(-, \underline{W}_{2}^{\wedge}\right)$ is exhaustive. Using the preliminary remark, we may replace $\Gamma\left(-, \underline{W}_{2}^{\wedge}\right)$ by $\Gamma\left(-, \underline{I}^{*} / \underline{I}^{*+1}\right)$, which coincides with $\operatorname{map}\left(\mathbb{G}_{m}^{\wedge *},(\mathrm{HZ} / 2) / \tau\right)$ according to [6, Theorem 2.1 , Lemma 2.7]. For this, we may establish the pullback square for $\mathrm{HF}_{\ell}$, which implies the pullback square for $\mathrm{H} \mathbb{Z}_{\ell}^{\wedge}$ and hence for $\mathrm{H} \mathbb{Z}_{\ell}^{\wedge} / 2 \simeq \mathrm{HZ} / 2$ since $2 \mid \ell$.

Finally, suppose that $\zeta_{\ell} \in X_{0}$. This yields $\tau \in \pi_{0,-1}\left(\mathrm{HF}_{\ell}\right)\left(X_{0}\right)$ given by the Bockstein on [ $\left.\zeta_{\ell}\right]$. The cofibres of $\tau$-powers yield a filtration of $\mathrm{HF}_{\ell}$ which pulls back to compatible filtrations on the $X_{i}$ s. The explicit construction of the motivic complexes [69, Theorem 3.9] shows that these filtrations are bounded, separated and exhaustive and have subquotients $\Gamma_{\mathrm{Zar}}\left(X_{i}, R^{m} \epsilon_{*} \mathbb{F}_{\ell}\right)$. Via the preliminary remark, the desired Cartesian square thus reduces to condition (1').

\section{Remark 4.4.}

- In all of our examples, the chain complexes in conditions (1') and (2) will be concentrated in a single degree.

- If $\bar{x}$ is the spectrum of a separably closed field, then $\Gamma_{\mathrm{Zar}}\left(\bar{x}, R^{m} \epsilon_{*} \mathbb{F}_{\ell}\right)=0$ for $m>0$, and similarly $\Gamma_{\text {rét }}(\bar{x}, \mathbb{Z})=0$.

- If the square (4.1) in Proposition 7 is co-Cartesian, then conditions (1) and (2) hold and (1') holds whenever $\zeta_{\ell} \in X_{0}$. Condition (3) is not necessary in general for the square to be co-Cartesian (consider, for example, any square comprising identity maps).

\subsection{Models for stable motivic homotopy types}

\subsubsection{Arithmetic preliminaries}

Lemma 4.5. Suppose $K$ is a global field with ring of integers $\mathcal{O}_{K}$ and put $U=\operatorname{Spec}\left(\mathcal{O}_{K}[1 / \ell]\right)$. If $\epsilon: U_{e} t \rightarrow U_{\mathrm{Zar}}$ is the change of topology functor, then

$$
R^{i} \epsilon_{*} \mu_{\ell} \simeq\left\{\begin{array}{ll}
\mu_{\ell} & i=0 \\
a_{\mathrm{Zar}} \mathcal{O}^{\times} / \ell & i=1 \\
a_{\text {rét }} \mathbb{Z} /(2, \ell) \oplus R & i=2 \\
a_{\text {rét }} \mathbb{Z} /(2, \ell) & i>2
\end{array} .\right.
$$

The sheaf $R$ is determined by the exact sequence

$$
0 \rightarrow R \rightarrow \bigoplus_{x \in \operatorname{Spec}\left(\mathcal{O}_{K}\right)^{(1)}} \mathbb{F}_{\ell} \rightarrow \mathbb{F}_{\ell} \oplus \bigoplus_{x \in U^{(1)}} i_{x *} \mathbb{F}_{\ell} \rightarrow 0
$$

Here the middle term is a constant sheaf, whereas the right-hand term is a sum of a constant sheaf and skyscraper sheaves, and the map is given by addition in the first component and restriction in the others.

Proof. From [52, Remark II.2.2] we can read off the isomorphisms

$$
R^{0} \epsilon_{*} \mathbb{G}_{m} \simeq \mathbb{G}_{m}, \quad R^{i} \epsilon_{*} \mathbb{G}_{m} \simeq 0 \text { for } i \text { odd, } \quad R^{i} \epsilon_{*} \mathbb{G}_{m} \simeq a_{\text {rét }} \mathbb{R}^{\times} \text {for } i \geq 4 \text { even }
$$

and the short exact sequence

$$
0 \rightarrow R^{2} \epsilon_{*} \mathbb{G}_{m} \rightarrow a_{\text {rét }} \mathbb{Z} / 2 \oplus \bigoplus_{x \in \operatorname{Spec}\left(\mathcal{O}_{K}\right)^{(1)}} \mathbb{Q} / \mathbb{Z} \rightarrow \mathbb{Q} / \mathbb{Z} \oplus \bigoplus_{x \in U^{(1)}} i_{x *} \mathbb{Q} / \mathbb{Z} \rightarrow 0
$$

For the exact sequence, recall $\operatorname{Br}\left(K_{v}\right)=\mathbb{Z} / 2$ if $v$ is a real place, $=0$ if $v$ is a complex place and $=\mathbb{Q} / \mathbb{Z}$ if $v$ is a non-Archimedean place $[67$, p. 163, 193]. Moreover, the kernel of the restriction map is precisely 
the sum over the non-Archimedean places missing in $U$. The Kummer short exact sequence

$$
0 \rightarrow \mu_{\ell} \rightarrow \mathbb{G}_{m} \stackrel{\ell}{\rightarrow} \mathbb{G}_{m} \rightarrow 0
$$

on $U_{\text {ét }}$ yields a long exact sequence for $R^{i} \epsilon_{*}$. Since $R^{i} \epsilon_{*} \mathbb{G}_{m}$ vanishes in odd degrees, $R^{i} \epsilon_{*} \mu_{\ell}$ is given by the kernel or cokernel of multiplication by $\ell$. This immediately yields the desired results for $i \neq 2,3$, and the snake lemma produces an exact sequence

$$
0 \rightarrow R^{2} \epsilon_{*} \mu_{\ell} \rightarrow a_{\text {rét }} \mathbb{Z} /(2, \ell) \oplus \bigoplus_{x \in \operatorname{Spec}\left(\mathcal{O}_{K}\right)^{(1)}} \mathbb{F}_{\ell} \stackrel{b}{\rightarrow} \mathbb{F}_{\ell} \oplus \bigoplus_{x \in U^{(1)}} i_{x *} \mathbb{F}_{\ell} \rightarrow R^{3} \epsilon_{*} \mu_{\ell} \rightarrow a_{\text {rét }} \mathbb{Z} /(2, \ell) \rightarrow 0
$$

Since $b$ is a surjection of Zariski sheaves, the result follows.

Corollary 8. Suppose Pic $(U)$ is uniquely $\ell$-divisible and $k(U)$ has a unique place of characteristic $\ell$. Then $H^{j}\left(U, R^{i} \epsilon_{*} \mu_{\ell}\right)=0$ for $j>0$ and

$$
H^{0}\left(U, R^{i} \epsilon_{*} \mu_{\ell}\right) \simeq \begin{cases}\mu_{\ell}(U) & i=0 \\ \mathcal{O}^{\times}(U) / \ell & i=1 \\ (\mathbb{Z} /(2, \ell))^{\operatorname{Sper}(K)} & i>1 .\end{cases}
$$

Proof. Since $\left.\mu_{\ell}\right|_{U_{Z a r}}$ is constant and constant sheaves are flasque, the claims for $i=0$ are clear. The claims about $a_{\text {rét }} \mathbb{Z} /(2, \ell)$ follow because $R(U) \simeq \operatorname{Sper}(k)$ is discrete. Since the Zariski cohomological dimension of $U$ is 1 , it remains to show that $H_{\mathrm{Zar}}^{0}\left(U, \mathbb{G}_{m} / \ell\right) \simeq \mathcal{O}^{\times}(U) / \ell, H_{\mathrm{Zar}}^{1}\left(U, \mathbb{G}_{m} / \ell\right)=0$, and $H_{\mathrm{Zar}}^{*}(U, R)=0$ for $*=0,1$. Using the short exact sequences $0 \rightarrow \mu_{\ell} \rightarrow \mathbb{G}_{m} \rightarrow \ell \mathbb{G}_{m} \rightarrow 0$ and $0 \rightarrow \ell \mathbb{G}_{m} \rightarrow \mathbb{G}_{m} \rightarrow \mathbb{G}_{m} / \ell \rightarrow 0$, the first two claims are equivalent to unique $\ell$-divisibility of $\operatorname{Pic}(U)$. The exact sequence defining $R$ is, in fact, a flasque resolution, so its $H^{0}$ and $H^{1}$ are given by the kernel and cokernel of the induced map on global sections. This induced map is an isomorphism as needed if and only if $\operatorname{Spec}\left(\mathcal{O}_{K}\right) \backslash U$ consists of precisely one point, which holds by assumption.

Lemma 4.6. Let $\ell$ be prime, $K$ a global field and $U \subset \operatorname{Spec}\left(\mathcal{O}_{K}\right)$ open. Let $H \subset \mathcal{O}^{\times}(U) / \ell$ be an arbitrary subgroup. There exist $x_{1}, \ldots, x_{n} \in U^{(1)}$ such that the restriction

$$
H \subset \mathcal{O}^{\times}(U) / \ell \rightarrow \prod_{i} k(x)^{\times} / \ell
$$

is an isomorphism. If $H$ is nontrivial, there exist infinitely many such choices.

Proof. First recall the following fact (see, e.g., [56, Exercise VI.1.2]): If $a \in \mathcal{O}(U)$ is an $\ell$ th power in $k(x)$ for all but finitely many $x \in U^{(1)}$, then $a$ is an $\ell$ th power.

If $H$ is nontrivial, pick $1 \neq a \in H$. Since $H$ is a $\mathbb{Z} / \ell$-vector space, we may write $H=\langle a\rangle \times H^{\prime}$, where $\langle a\rangle \simeq \mathbb{Z} / \ell$ is the subgroup generated by $a$. By the above fact, there exists $x \in U^{(1)}$ such that the image of $a$ in $k(x)^{\times} / \ell$ is nonzero and in fact infinitely many choices of $x$. Since $k(x)$ is finite, $k(x)^{\times} / \ell \simeq \mathbb{Z} / \ell \simeq\langle a\rangle$. We are thus reduced to proving the result for $H^{\prime}$ and conclude by induction since $\mathcal{O}^{\times}(U) / \ell$ is finite according to Dirichlet's unit theorem [56, Corollary 11.7].

In [30], Gras introduced the narrow tame kernel $K_{2}^{+}\left(\mathcal{O}_{F}\right)$ as the subgroup of $K_{2}\left(\mathcal{O}_{F}\right)$ where the regular symbols on all of the real embeddings of $F$ vanish; that is, there is an exact sequence

$$
0 \rightarrow K_{2}^{+}\left(\mathcal{O}_{F}\right) \rightarrow K_{2}\left(\mathcal{O}_{F}\right) \rightarrow \bigoplus^{r} \mathbb{Z} / 2 \rightarrow 0
$$

We refer to [31, Definition 7.8.1] for the arithmetic notion of $\ell$-regular number fields.

Definition 9. Let $\ell$ be a prime number. A number field $F$ is called $\ell$-regular if the $\ell$-Sylow subgroup of the narrow tame kernel $K_{2}^{+}\left(\mathcal{O}_{F}\right)$ is trivial. 
See [30], [32], [60], [15] for complementary results about these families of number fields. For example, the field of rational numbers $\mathbb{Q}$ is $\ell$-regular for every prime $\ell$, and $\mathbb{Q}\left(\zeta_{\ell}\right)$ is $\ell$-regular if $\ell$ is a regular prime number in the sense of Kummer [73]. In [68], Siegel conjectured there are infinitely many regular prime numbers.

We have the following explicit characterisation of $\ell$-regular number fields.

Proposition 10. Let $F$ be a number field. We write $\mathcal{O}_{F}^{\prime}$ for the ring of $\ell$-integers $\mathcal{O}_{F}[1 / \ell]$.

1. $F$ is 2-regular if and only if the prime ideal (2) does not split in $F / \mathbb{Q}$ and the narrow Picard group Pic $_{+}\left(\mathcal{O}_{F}^{\prime}\right)$ has odd order.

2. Let $\ell$ be an odd prime number and assume $\mu_{\ell} \subset F$. Then $F$ is $\ell$-regular if and only if the prime ideal $(\ell)$ does not split in $F / \mathbb{Q}$ and the $\ell$-Sylow subgroup of the Picard group Pic $\left(\mathcal{O}_{F}^{\prime}\right)$ is trivial.

3. Let $\ell$ be an odd prime number. Assume $\mu_{\ell} \not \subset F$ and $F$ contains the maximal real subfield of $\mathbb{Q}\left(\zeta_{\ell}\right)$. Then $F$ is $\ell$-regular if and only if the prime ideals above $(\ell)$ in $F$ do not split in the quadratic extension $F\left(\zeta_{\ell}\right) / F$ and the $\ell$-Sylow subgroups of the Picard groups $\operatorname{Pic}\left(\mathcal{O}_{F}\right)$ and $\operatorname{Pic}\left(\mathcal{O}_{F\left(\zeta_{\ell}\right)}\right)$ are isomorphic.

Proof. This is a reformulation of [30, Corollary on pp. 328-329]. See also [60, Proposition 2.2] when $\ell=2$.

For further reference, we recall that a commutative square of abelian groups

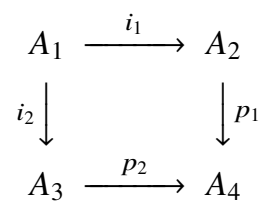

is called bi-Cartesian if it is a pullback when viewed as a commutative square of spectra.

\subsubsection{Stable motivic homotopy types of 2-regular number fields}

Theorem 4.7. Suppose $F$ is a 2-regular number field with $r$ real and c pairs of complex embeddings. Let $x, y_{1}, \ldots, y_{c} \in \operatorname{Spec}\left(\mathcal{O}_{F}^{\prime}\right)$ be closed points.

1. There is a canonical commutative square in $\mathcal{C M}$

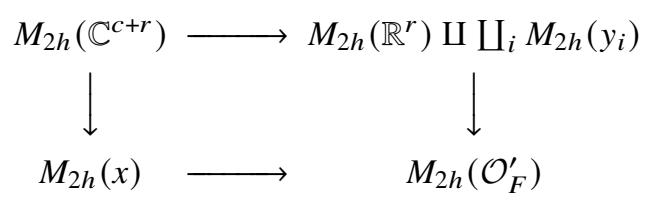

2. The square in (1) is a pushout if and only if there is a naturally induced isomorphism

$$
\left(\mathcal{O}_{F}^{\prime}\right)^{\times} / 2 \simeq\left(\mathbb{R}^{\times} / 2\right)^{r} \times k(x)^{\times} / 2 \times \prod_{i} k\left(y_{i}\right)^{\times} / 2 \quad\left(\simeq(\mathbb{Z} / 2)^{1+r+c}\right) .
$$

3. There exist infinitely many choices of $x, y_{1}, \ldots, y_{c}$ such that the map in (2) is an isomorphism.

Proof. To simplify notation, throughout this proof we put $M:=M_{2 h}$.

(1) For $z \in \operatorname{Spec}\left(\mathcal{O}_{F}^{\prime}\right)$ and $\alpha: K \hookrightarrow \mathbb{C}$, Lemma 4.2 furnishes a map $f_{z, \alpha}: M(\mathbb{C}) \rightarrow M(z)$ and a homotopy between $M(\mathbb{C}) \rightarrow M(z) \rightarrow M\left(\mathcal{O}_{F}^{\prime}\right)$, and the map $M(\mathbb{C}) \rightarrow M\left(\mathcal{O}_{F}^{\prime}\right)$ induced by $\alpha$. In (1), the bottom and right-hand maps are the canonical ones. Write $\alpha_{1}, \bar{\alpha}_{1}, \ldots, \alpha_{c}, \bar{\alpha}_{c}, \beta_{1}, \ldots, \beta_{r}$ for the complex and real embeddings. Let $\alpha_{c+i}=\iota \circ \beta_{i}$, where $\iota: \mathbb{R} \rightarrow \mathbb{C}$ is the canonical embedding. The left-hand map is $f_{x, \alpha_{i}}$ on component $i$. The top map is $f_{y_{i}, \alpha_{i}}$ on the $i$ th component if $i \leq c$ and induced by $\iota$ on the remaining components. In all cases, the induced composite map $M(\mathbb{C}) \rightarrow M\left(\mathcal{O}_{F}^{\prime}\right)$ is either equal or homotopic to the map induced by $\alpha_{i}$. Thus, the square commutes. 
(2) We use the criterion from Proposition 7. Condition (3) holds since the fields are finitely generated. For condition (2), the square

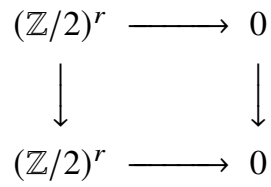

is clearly bi-Cartesian because the map $\mathcal{O}_{F}^{\prime} \rightarrow \mathbb{R}^{r}$ induces an isomorphism on real spectra.

Next we check condition (1'). Owing to [15, Proposition 2.1(5)] the 2-regularity assumption implies $\operatorname{Pic}\left(\mathcal{O}_{F}^{\prime}\right)$ has odd order, so it is uniquely 2-divisible, and $F$ has only one place of characteristic 2 . Thus, Corollary 8 applies and it remains to check the bicartesianess of three squares. The first one is

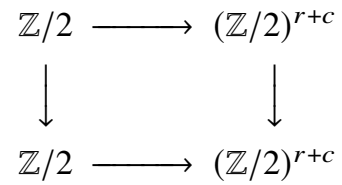

Observe that if $X, Y$ are connected schemes and $f: M(X) \rightarrow M(Y)$ is any map in $\mathcal{C M}_{\mathbb{Z}[1 / 2]}$, then $f^{*}: H^{0}(Y, \mathbb{Z} / 2) \rightarrow H^{0}(X, \mathbb{Z} / 2)$ is an isomorphism. Indeed, this reduces to the case of the structure map $M(X) \rightarrow M(\mathbb{Z}[1 / 2])$, where it is obvious. Thus, the square for $m=0$ is bi-Cartesian because the vertical maps are isomorphisms. When $m=2$, the square is the same as in condition (2) above and hence it is bi-Cartesian. The remaining square for $m=1$ takes the form

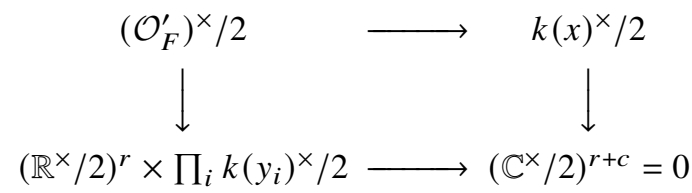

Since the inclusion of abelian groups into spectra preserves finite products, this square is bi-Cartesian if and only if the stated condition holds.

(3) Dirichlet's unit theorem [56, Corollary 11.7] implies $\left(\mathcal{O}_{F}^{\prime}\right)^{\times} \simeq \mu\left(\mathcal{O}_{F}^{\prime}\right) \times \mathbb{Z}^{r+c}$; here $\mu\left(\mathcal{O}_{F}^{\prime}\right)$ is the finite abelian group of roots of unity in $\mathcal{O}_{F}^{\prime}$. It is cyclic, being a finite multiplicative subgroup of a field, and since $\{ \pm 1\} \in \mu\left(\mathcal{O}_{F}^{\prime}\right)$, the group has even order. It follows that $\left(\mathcal{O}_{F}^{\prime}\right)^{\times} / 2 \simeq \mathbb{Z} / 2 \times(\mathbb{Z} / 2)^{r+c}$. Moreover, 2-regularity implies the naturally induced map $\left(\mathcal{O}_{F}^{\prime}\right)^{\times} / 2 \rightarrow\left(\mathbb{R}^{\times} / 2\right)^{r} \simeq(\mathbb{Z} / 2)^{r}$ is surjective [15, Proposition 2.1(5)]; we write $U_{+} \simeq(\mathbb{Z} / 2)^{1+c}$ for its kernel. The condition in part (2) holds if and only if the induced map $U_{+} \rightarrow k(x)^{\times} / 2 \times \prod_{i} k\left(y_{i}\right)^{\times} / 2$ is an isomorphism. Lemma 4.6 implies the latter is true for infinitely many choices of $x, y_{i}$.

Remark 4.8. As in Examples 3.3 and 3.4, Theorem 4.7(1) implies similar pushout squares with respect to completions at $2, h$ and with respect to periodisations at $\rho, \eta$. For example, we have a pushout square in $\mathcal{C M}$

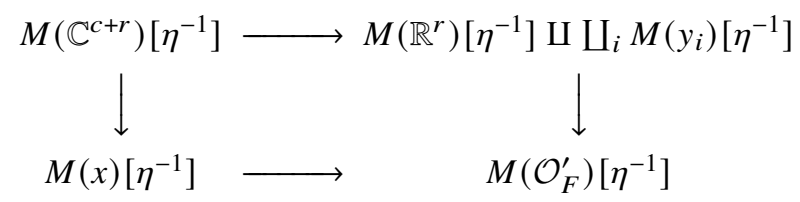

Remark 4.9. The various embeddings $\alpha_{i}: K \rightarrow \mathbb{C}$ differ by automorphisms of $\mathbb{C}$. It follows that one may choose the maps $f_{x, \alpha_{i}}$ to be of the form $\sigma_{i} \circ f_{x, \alpha_{1}}$. Thus, applying an automorphism of $M_{2 h}\left(\mathbb{C}^{r+s}\right)$ in the square of Theorem 4.7, we may assume that all of the left-hand vertical maps $M_{2 h}(\mathbb{C}) \rightarrow M_{2 h}(x)$ are 
the same. The square being a pushout now is equivalent to saying that there are lifts of $M_{2 h}(x), M_{2 h}\left(y_{i}\right)$ to $\mathcal{C M}_{M_{2 h}(\mathbb{C}) /}$ and an equivalence

$$
M_{2 h}\left(\mathcal{O}_{F}^{\prime}\right) \simeq \bigvee^{r} M_{2 h}(\mathbb{R}) \vee M_{2 h}(x) \vee \bigvee^{c} M_{2 h}\left(y_{i}\right)
$$

Here $\vee$ denotes the coproduct in $\mathcal{C M}_{M(\mathbb{C}) /}$.

Example 4.10. When $F=\mathbb{Q}$, we consider $\mathbb{Z}[1 / 2]^{\times} \simeq\{ \pm 1\} \times\left\{(1 / 2)^{n}\right\}$ and $\mathbb{Z}[1 / 2]^{\times} / 2 \simeq \mathbb{Z} / 2\{-1,2\}$. Here $\mathbb{Z} / 2\{2\}$ is the kernel of the surjection $\mathbb{Z}[1 / 2]^{\times} / 2 \rightarrow \mathbb{R}^{\times} / 2$. We need to find a closed point $x \in \operatorname{Spec}(\mathbb{Z}[1 / 2])$ such that 2 is not a square in $k(x)$. This holds when $k(x)=\operatorname{Spec}\left(\mathbb{F}_{q}\right)$, where $q \equiv \pm 3 \bmod 8$. In particular, the canonical map

$$
\mathcal{S H}(\mathbb{Z}[1 / 2])_{2}^{\wedge \text { cell }} \rightarrow \mathcal{S H}(\mathbb{R})_{2}^{\wedge \text { cell }} \times_{\mathcal{S H}(\mathbb{C})_{2}^{\wedge \text { cell }}} \mathcal{S H}\left(\mathbb{F}_{3}\right)_{2}^{\wedge \text { cell }}
$$

is fully faithful. To deduce Theorem 1.2 from the introduction, let $\mathcal{E} \in \mathcal{S H}(\mathbb{Z}[1 / 2])_{2}^{\wedge \text { cell }}$ and compute $\operatorname{map}(\mathbf{1}, \mathcal{E})$ using the above square.

\subsubsection{Stable motivic homotopy types of $\ell$-regular number fields}

Theorem 4.11. Let $F$ be a number field with c pairs of complex embeddings and $\ell$ be an odd prime number. Suppose $F$ is $\ell$-regular and $\mu_{\ell} \subset F$. Let $x, y_{1}, \ldots, y_{c} \in \operatorname{Spec}\left(\mathcal{O}_{F}^{\prime}\right)$ be closed points.

1. There is a canonical commutative square in $\mathrm{CM}$

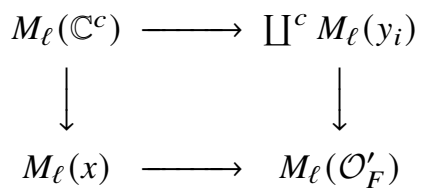

2. The square is a pushout if and only if there is a naturally induced isomorphism

$$
\left(\mathcal{O}_{F}^{\prime}\right)^{\times} / \ell \simeq k(x)^{\times} / \ell \times \prod_{i} k\left(y_{i}\right)^{\times} / \ell \quad\left(\simeq\left(\mathbb{F}_{\ell}\right)^{1+c}\right) .
$$

3. There exist infinitely many choices of $x, y_{1}, \ldots, y_{c}$ satisfying (2).

Proof. The proof is essentially the same as that of Theorem 4.7. The maps $x, y_{i} \rightarrow \operatorname{Spec}\left(\mathcal{O}_{F}^{\prime}\right)$ together with choices of embeddings of $K$ into $\mathbb{C}$ induce, via Lemma 4.2, the maps $M_{\ell}(\mathbb{C}) \rightarrow M_{\ell}(x), M_{\ell}\left(y_{i}\right)$ in the commutative square. One verifies, using Corollary 8 and $\mathbb{Z} /(2, \ell)=0$, that condition (1') of Proposition 7 reduces to the condition stated in (2). The other conditions hold trivially; since $K$ contains a primitive $\ell$ th root of unity, the real spectrum $\operatorname{Sper}\left(\mathcal{O}_{F}^{\prime}\right)=\emptyset$. The existence of infinitely many choices in (3) follows from Lemma 4.6.

Remark 4.12. Arguing as in Remark 4.9, we find that there are lifts of $M_{\ell}(x), M_{\ell}\left(y_{i}\right)$ to $\mathcal{C M}_{M_{\ell}(\mathbb{C}) /}$ and an equivalence

$$
M_{\ell}\left(\mathcal{O}_{F}^{\prime}\right) \simeq \bigvee^{c} M_{\ell}\left(y_{i}\right) \vee M_{\ell}(x)
$$

Example 4.13. Theorem 4.11 applies to $F=\mathbb{Q}\left(\zeta_{\ell}\right)$ if $\ell$ is regular - we note that $(\ell)$ is totally ramified in $F$ and $K_{2}\left(\mathbb{Z}\left[\zeta_{\ell}\right]\right) / \ell \equiv \mu_{\ell} \otimes \operatorname{Pic}\left(\mathbb{Z}\left[\zeta_{\ell}\right]\right)$. In this case, $\mathcal{O}_{F}^{\prime}=\mathbb{Z}\left[1 / \ell, \zeta_{\ell}\right]$ and $k(x)=\mathbb{F}_{p}$, where $p$ is a prime number which is congruent to $1 \bmod \ell$ but is not congruent to $1 \bmod \ell^{2}$ by [24, Example 1.9]. 
Theorem 4.14. Let $\ell$ be an odd regular prime and $p \neq \ell$ a prime number. There is a commutative square in $\mathcal{C M}_{\mathbb{Z}[1 / \ell]}$

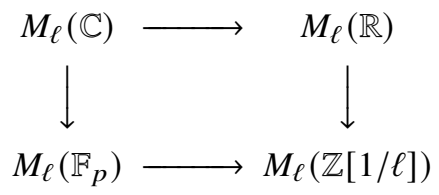

The square is a pushout if $p$ generates the multiplicative group of units $\left(\mathbb{Z} / \ell^{2}\right)^{\times}$.

Proof. We get the square from Lemma 4.2 and proceed by verifying the conditions in Proposition 7. Since $\mathbb{Z}[1 / \ell]$ has a unique real embedding, condition (2) holds. Condition (3) is vacuous. Next we verify condition (1). Let us write $\Gamma\left(X, \mathbb{F}_{\ell}(i)\right)$ for the motivic complex and $\Gamma_{e ́ t}\left(X, \mathbb{F}_{\ell}(i)\right) \simeq \Gamma_{e ́ t}\left(X, \mu_{\ell}^{\otimes i}\right)$ for its étale version. If $A=\mathbb{Z}\left[1 / \ell, \zeta_{\ell}\right]$, then $H_{e ́ t}^{0}\left(A, \mathbb{F}_{\ell}\right)=\mathbb{F}_{\ell}, H_{e ́ t}^{1}\left(A, \mathbb{F}_{\ell}\right)=A^{\times} / \ell$ and $H_{e ́ t}^{*}\left(A, \mathbb{F}_{\ell}\right)=0$ else; see [52, Remark II.2.2]. Corollary 8 implies that $\Gamma\left(A, \mathbb{F}_{\ell}(i)\right) \simeq \Gamma_{e ́ t}\left(A, \mathbb{F}_{\ell}(i)\right)_{\geq-i}$. A transfer argument shows $\Gamma\left(\mathbb{Z}[1 / \ell], \mathbb{F}_{\ell}(i)\right)$ is a summand of $\Gamma\left(A, \mathbb{F}_{\ell}(i)\right)$, and similarly for $\Gamma_{e ́ t}$. We deduce the equivalence

$$
\Gamma\left(\mathbb{Z}[1 / \ell], \mathbb{F}_{\ell}(i)\right) \simeq \Gamma_{e ́ t}\left(\mathbb{Z}[1 / \ell], \mathbb{F}_{\ell}(i)\right)_{\geq-i} .
$$

The same is true for $\mathbb{C}, \mathbb{R}, \mathbb{F}_{p}$ since they are Nisnevich local. Consequently, condition (1) will hold if the square

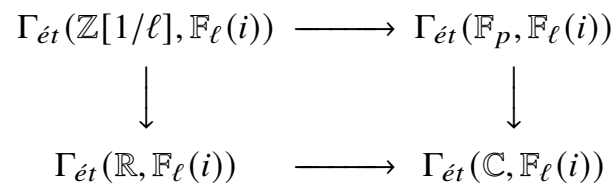

is Cartesian and the maps

$$
H_{e ̂ t}^{i}\left(\mathbb{R}, \mathbb{F}_{\ell}(i)\right) \oplus H_{e ́ t}^{i}\left(\mathbb{F}_{p}, \mathbb{F}_{\ell}(i)\right) \rightarrow H_{e ́ t}^{i}\left(\mathbb{C}, \mathbb{F}_{\ell}(i)\right)
$$

are surjective for every $i$. The first condition holds by [24, Theorem 2.1]. The second condition is vacuous when $i>0$ and easily verified for $i=0$.

Remark 4.15. By adjoining an $\ell$ th root of unity, one obtains the commutative square

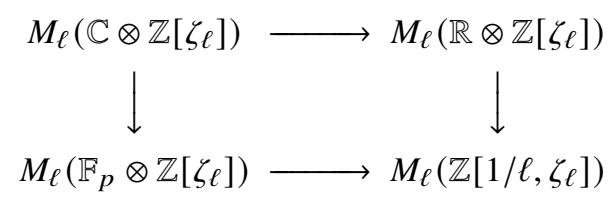

This induces a Cartesian square in étale cohomology but not in motivic cohomology (since, e.g., the group $H^{1,0}\left(\mathbb{Z}\left[1 / \ell, \zeta_{\ell}\right], \mathbb{F}_{\ell}\right)=0$ but the corresponding map on $H^{0,0}$ is not surjective).

\subsubsection{Relation to étale homotopy types}

Corresponding to the squares in Theorems 4.7, 4.11 and 4.14, there are analogous squares of étale homotopy types; in fact, Lemma 4.2, the only nonformal input in the construction of the said squares, also holds for étale homotopy types. Due to the equivalence

$$
\mathrm{H}_{e ́ t} \mathbb{Z} / \ell^{n} \simeq \mathrm{H} \mathbb{Z} / \ell^{n}\left[(\tau)^{-1}\right] \in \mathcal{S H}(S)_{\ell}^{\wedge \text { cell }}
$$

from, for example, [9, Theorem 7.4], our co-Cartesian squares in $\mathcal{C M}_{\mathbb{Z}[1 / \ell]}$ induce Cartesian squares in étale cohomology with $\mathbb{Z} / \ell^{n}(i)$-coefficients. By Dwyer-Friedlander [24, Theorem 2.1] 
[23, pp. 144-145], the resulting squares of étale homotopy types become pushouts after appropriate homological localisation.

By analysing the proof of Theorem 4.14, one sees that condition (1) in Proposition 7 is satisfied if the following hold:

○ $\operatorname{dim} X_{0} \leq 1, \operatorname{dim} X_{n}=0$ else.

- The induced square of étale cohomology with $\mathbb{F}_{\ell}(i)$-coefficients is Cartesian.

- The induced square of Zariski cohomology with $\mathbb{F}_{\ell}$-coefficients is Cartesian.

○ The group $H_{\mathrm{Zar}}^{1}\left(X_{0}, R^{i} \epsilon_{*} \mathbb{F}_{\ell}(i)\right)=0$ for $i \geq 0$.

\section{Applications to slice completeness and universal motivic invariants}

We apply the results in Section 4 to show slice completeness and compute the endomorphism ring of the motivic sphere over regular number rings. Our completeness result for Voevodsky's slice filtration [72] is motivated by applications such as motivic generalisations of Thomason's étale descent theorem for algebraic $K$-theory in [25] and [9], convergence of the slice filtration [45], the solution of Milnor's conjecture on quadratic forms in [61], computations of universal motivic invariants in [64] and of hermitian $K$-groups in [43].

For the standard nomenclature associated with the slice filtration, such as the effective covers $f_{q}$ and the effective cocovers $f^{q}$, and the slice completion sc we refer to [63, §3, (3.1), (3.3), (3.10)]. Let $\mathcal{S H}(S)_{\geq 0}$ denote the connective motivic spectra with respect to the homotopy $t$-structure on $\mathcal{S H}(S)$ [36, §2.1]. The notion of a cell presentation of finite type is defined in [64, §3.3]. We shall say that a completeness property requiring a map $E \rightarrow F$ to be an equivalence holds 'on homotopy' if $\pi_{* *} E \rightarrow \pi_{* *} F$ is an isomorphism.

Proposition 11. Suppose $F$ is a 2-regular number field and set $\mathcal{O}_{F}^{\prime}:=\mathcal{O}_{F}[1 / 2]$.

1. Let $\mathcal{E}_{\bullet} \in \mathcal{S H}\left(\mathcal{O}_{F}^{\prime}\right)_{2}^{\wedge c e l l}$ be a tower such that $\lim _{n} p_{i}^{*}\left(\mathcal{E}_{n}\right) \simeq 0$, where $p_{i}^{*}$ denotes the pullback to any of the fields in Theorem 4.7(1). Then $\lim _{n} \mathcal{E}_{n} \simeq 0$ is contractible.

2. If $\mathcal{E} \in \mathcal{S H}\left(\mathcal{O}_{F}^{\prime}\right)^{\text {veff }} \cap \mathcal{S H}\left(\mathcal{O}_{F}^{\prime}\right)^{\text {cell }}$ is cellular and very effective, then $\mathcal{E} / 2$ is $\eta$-complete on homotopy.

3. Let $\mathcal{E} \in \mathcal{S H}\left(\mathcal{O}_{F}^{\prime}\right)_{\geq 0} \cap \mathcal{S H}\left(\mathcal{O}_{F}^{\prime}\right)^{\text {cell }}$ and assume the slices of $\mathcal{E}$ are cellular and stable under base change. Then there is an isomorphism

$$
\pi_{*, *}\left(\lim _{n} f^{n}(\mathcal{E}) /(2, \rho)\right) \simeq \pi_{*, *}(\mathcal{E} /(2, \rho)) .
$$

4. Let $\mathcal{E} \in \mathcal{S H}\left(\mathcal{O}_{F}^{\prime}\right)^{\text {eff }} \cap \mathcal{S H}\left(\mathcal{O}_{F}^{\prime}\right)^{\text {cell }}$ be cellular and effective. Assume $\mathcal{E} / 2$ has a $\mathbb{Z}_{(2)}$-cell presentation of finite type and its slices are cellular and stable under base change. Then $\mathcal{E} /(2, \eta)$ is slice complete on homotopy and

$$
\pi_{*, *}\left(\operatorname{sc}(\mathcal{E})_{2}^{\wedge}\right) \simeq \pi_{*, *}\left(\mathcal{E}_{2, \eta}^{\wedge}\right)
$$

In particular, there is an isomorphism

$$
\pi_{*, *}\left(\operatorname{sc}(\mathbf{1})_{2}^{\wedge}\right) \simeq \pi_{*, *}\left(\mathbf{1}_{2}^{\wedge}\right)
$$

Proof. (1) Let $I=\{* \rightarrow * \leftarrow *\}$ be the category so that $\lim _{I}$ means pullback. For all $X \in \mathcal{S H}\left(\mathcal{O}_{F}^{\prime}\right)_{2}^{\wedge \text { cell }}$ we compute

$$
\begin{aligned}
& \operatorname{Map}\left(X, \lim _{n} \mathcal{E}_{n}\right) \simeq \lim _{n} \operatorname{Map}\left(X, \mathcal{E}_{n}\right) \simeq \lim _{n} \lim _{i \in I} \operatorname{Map}\left(p_{i}^{*}(X), p_{i}^{*}\left(\mathcal{E}_{n}\right)\right) \\
& \simeq \lim _{i} \lim _{n} \operatorname{Map}\left(p_{i}^{*}(X), p_{i}^{*}\left(\mathcal{E}_{n}\right)\right) \simeq \lim _{i} \operatorname{Map}\left(p_{i}(X), \lim _{n} p_{i}^{*}\left(\mathcal{E}_{n}\right)\right) \simeq 0 .
\end{aligned}
$$

The result follows. 
(2) Recall that $\mathcal{E}$ is $\eta$-complete if and only if

$$
\lim \left[\cdots \stackrel{\eta}{\rightarrow} \Sigma^{2,2} \mathcal{E} \stackrel{\eta}{\rightarrow} \Sigma^{1,1} \mathcal{E} \stackrel{\eta}{\rightarrow} \mathcal{E}\right] \simeq 0
$$

Thus, by (1) it suffices to check $p_{i}^{*}(\mathcal{E} / 2)$ is $\eta$-complete for each $i$, which holds by [10, Theorem 5.1].

(3) The claim holds if and only if $\lim _{n} f_{n}(\mathcal{E}) /(2, \rho) \simeq 0$ on homotopy groups or, equivalently, when computed in $\mathcal{S H}\left(\mathcal{O}_{F}^{\prime}\right)^{\text {cell }}$. The assumptions imply $f_{n}(\mathcal{E}) \in \mathcal{S H}\left(\mathcal{O}_{F}^{\prime}\right)^{\text {cell }}$ and $p_{i}^{*} f_{n} \mathcal{E} \simeq f_{n} p_{i}^{*} \mathcal{E}$. Hence, by (1) it suffices to note that $\lim _{n} f^{n}\left(p_{i}^{*} \mathcal{E}\right) /(2, \rho) \simeq p_{i}^{*}(\mathcal{E}) /(2, \rho)$ owing to [10, Proposition 5.2].

(4) For the first statement we need to prove $\lim _{n} f_{n}(\mathcal{E} /(2, \eta)) \simeq 0$ on homotopy groups. As in (3), this reduces to the same statement over fields, which holds by [64, Proposition 3.49]. For the second statement we need to $\operatorname{show} \operatorname{sc}(\mathcal{E} / 2) \simeq \mathcal{E}_{\eta}^{\wedge} / 2$, which holds by the proof of [64, Lemma 3.13]: $\operatorname{sc}(\mathcal{E} / 2)$ is $\eta$-complete since $\mathcal{E} / 2$ is effective, and $\operatorname{sc}(\mathcal{E} / 2) / \eta \simeq \operatorname{sc}(\mathcal{E} /(2, \eta)) \simeq \mathcal{E} /(2, \eta)$ - the first equivalence holds by inspection of the slices. The final statement follows since the slices of $\mathbf{1}_{(2)}$ over $\mathcal{O}_{F}^{\prime}$ are known and have the desired properties by [64, Remark 2.2, Theorem 2.12].

Remark 5.1. We expect that analogs of Proposition 11 hold over more general base schemes. Moreover, we expect that these results hold without the qualification 'on homotopy'. Both shortcomings are a result of our specific technique for accessing global sections of cellular spectra over arithmetic base schemes.

Recall that any unit $a \in \mathcal{O}(S)^{\times}$gives rise to a map $[a]: \mathbf{1} \rightarrow S^{1,1} \in \mathcal{S H}(S)$ and hence an element

$$
\langle a\rangle:=1+\eta[a] \in \pi_{0,0}\left(\mathbf{1}_{S}\right)
$$

This turns $\pi_{0,0}(\mathbf{1})$ into an $\mathbb{Z}\left[\mathcal{O}(S)^{\times}\right]$-algebra. We made use of the algebra structure in the formulation of Theorem 1.4 for $\mathbb{Z}[1 / 2]$. The generalisation to 2-regular number rings takes the following form.

Theorem 5.2. Suppose $F$ is a 2-regular number field with $r$ real embeddings and c pairs of complex embeddings. For the endomorphism ring of the motivic sphere over the base scheme $\mathcal{O}_{F}^{\prime}:=\mathcal{O}_{F}[1 / 2]$ there is an isomorphism of $\mathbb{Z}\left[\left(\mathcal{O}_{F}^{\prime}\right)^{\times}\right]$-algebras

$$
\pi_{0,0}\left(\mathbf{1}_{\mathcal{O}_{F}^{\prime}}\right) \otimes \mathbb{Z}_{(2)} \simeq \mathrm{GW}\left(\mathcal{O}_{F}^{\prime}\right) \otimes \mathbb{Z}_{(2)}
$$

induced by the unit map $\mathbf{1} \rightarrow \mathrm{KO}$. Moreover, we have the vanishing result

$$
\pi_{*, 0}\left(\mathbf{1}_{\mathcal{O}_{F}^{\prime}}\right) \otimes \mathbb{Z}_{(2)}=0 . \quad \text { for } \quad *<0
$$

Proof. The presentation of Grothendieck-Witt rings of fields of characteristic $\neq 2$ by generators and relations given in $[44$, Theorem 4.1$]$ implies there are $\mathbb{Z}\left[\left(\mathcal{O}_{F}^{\prime}\right)^{\times}\right]$-algebra isomorphisms

$$
\mathrm{GW}(\mathbb{R}) \simeq \mathbb{Z} \oplus \mathbb{Z}\{\langle-1\rangle\}, \mathrm{GW}(\mathbb{C}) \simeq \mathbb{Z}, \mathrm{GW}\left(\mathbb{F}_{q}\right) \simeq \mathbb{Z} \oplus \mathbb{Z} / 2
$$

In the isomorphism for $\mathrm{GW}\left(\mathbb{F}_{q}\right)$, the right-hand side has trivial multiplication on the square class group $\mathbb{F}_{q}^{\times} /\left(\mathbb{F}_{q}^{\times}\right)^{2} \simeq \mathbb{Z} / 2$. As such, every $n$-dimensional form in $\mathrm{GW}\left(\mathbb{F}_{q}\right)$ can be written as either $n\langle 1\rangle$ or $(n-1)\langle 1\rangle \oplus\langle a\rangle$, where $a$ is a nonsquare element in $\mathbb{F}_{q}^{\times}$(we may choose $a=-1$ if and only if $q \equiv 3 \bmod 4$ ). Moreover, by [15, Proposition 2.1(7)] and the proof of [13, Theorem 5.8], one deduces the $\mathbb{Z}\left[\left(\mathcal{O}_{F}^{\prime}\right)^{\times}\right]$-algebra isomorphism

$$
\mathrm{GW}\left(\mathcal{O}_{F}^{\prime}\right) \simeq \mathbb{Z}^{1+r} \oplus(\mathbb{Z} / 2)^{1+c}
$$


Thus, for the closed points $x, y_{1}, \ldots, y_{c} \in \operatorname{Spec}\left(\mathcal{O}_{F}^{\prime}\right)$ in the notation of Theorem 4.7 , there is a pullback square of $\mathbb{Z}\left[\left(\mathcal{O}_{F}^{\prime}\right)^{\times}\right]$-algebras

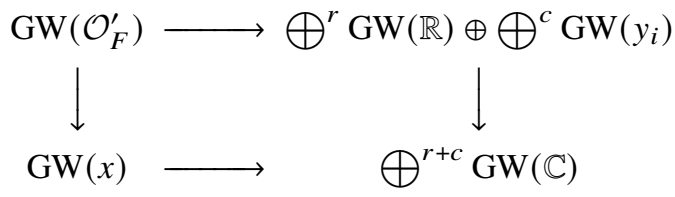

The Grothendieck-Witt rings appearing in (5.1) are quotients of $\mathbb{Z}\left[\left(\mathcal{O}_{F}^{\prime}\right)^{\times}\right]$. Thus, the maps in (5.1) are unique as $\mathbb{Z}\left[\left(\mathcal{O}_{F}^{\prime}\right)^{\times}\right]$-algebra maps. Since 2 -adic completion is exact on finitely generated abelian groups, this square remains Cartesian after 2-adic completion.

Consider the long exact sequence of homotopy groups associated with the pullback square

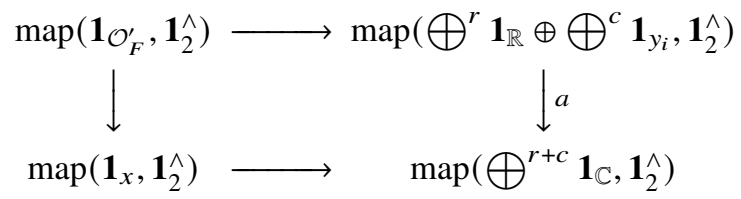

We have $\pi_{*, 0}\left(\mathbf{1}_{2}^{\wedge}\right)(\mathbb{C}) \simeq\left(\pi_{*}^{s}\right)_{2}^{\wedge}$ by [46, Corollary 2]. It follows that the right vertical map in (5.2) is surjective on homotopy groups. Indeed, recall that $\mathcal{S} \mathcal{H}^{f i n}$ is the initial stable symmetric monoidal $\infty$-category according to [16, Theorem 3.1]. Thus, for any symmetric monoidal stable $\infty$-category $\mathcal{C}$ and symmetric monoidal functor $F: \mathcal{C} \rightarrow \mathcal{S H}(\mathbb{C})_{2}^{\wedge}$, there exists a factorisation

$$
\left(\pi_{*}^{s}\right)_{2}^{\wedge} \rightarrow \pi_{*}\left(c_{2}^{\wedge}\right) \stackrel{F}{\rightarrow} \pi_{*, 0}\left(\left(\mathbf{1}_{\mathbb{C}}\right)_{2}^{\wedge}\right)
$$

and the composite is surjective by Levine's result. Thus, using [55, Corollary 6.43], we deduce the pullback square of rings

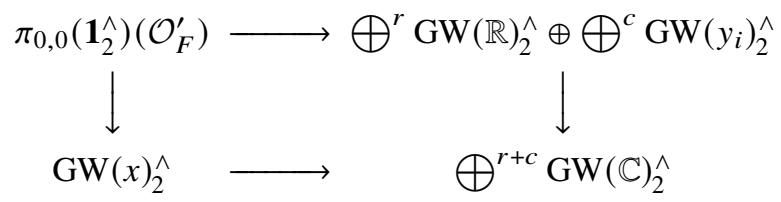

Note that (5.3) comes from a diagram in $\mathcal{C M}_{\mathcal{O}_{F}^{\prime}}$. Hence, the maps in (5.3) are $\pi_{0,0}\left(\mathbf{1}_{2}^{\wedge}\right)\left(\mathcal{O}_{F}^{\prime}\right)$-algebra maps, so a fortiori $\mathbb{Z}\left[\left(\mathcal{O}_{F}^{\prime}\right)^{\times}\right]$-algebra maps. The Grothendieck-Witt rings in (5.3) are quotients of $\mathbb{Z}\left[\left(\mathcal{O}_{F}^{\prime}\right)^{\times}\right]_{2}^{\wedge}$; thus, the lower horizontal and right-hand vertical maps in (5.3) are unique $\mathbb{Z}\left[\left(\mathcal{O}_{F}^{\prime}\right)^{\times}\right]$algebra maps. Thus, (5.3) is the 2 -adic completion of (5.2) and there is an isomorphism of $\mathbb{Z}\left[\left(\mathcal{O}_{F}^{\prime}\right)^{\times}\right]$algebras

$$
\pi_{0,0}\left(\mathbf{1}_{2}^{\wedge}\right)\left(\mathcal{O}_{F}^{\prime}\right) \simeq \operatorname{GW}\left(\mathcal{O}_{F}^{\prime}\right)_{2}^{\wedge}
$$

There is a similar pullback square for $\pi_{1,0}(-) \otimes \mathbb{Q}$. Since the vanishing $\pi_{1,0}\left(\mathbf{1}_{2}^{\wedge}\right)(k) \otimes \mathbb{Q}=0$ holds for $k=\mathbb{R}\left[21\right.$, Figure 4], $k=\mathbb{C}\left[46\right.$, Corollary 2] and $k=\mathbb{F}_{q}[75$, Theorem 1.3], we deduce the vanishing

$$
\pi_{1,0}\left(\mathbf{1}_{2}^{\wedge}\right)\left(\mathcal{O}_{F}^{\prime}\right) \otimes \mathbb{Q}=0
$$


Inserted into the fracture square long exact sequence we get a pullback square of $\mathbb{Z}\left[\left(\mathcal{O}_{F}^{\prime}\right)^{\times}\right]$-algebras

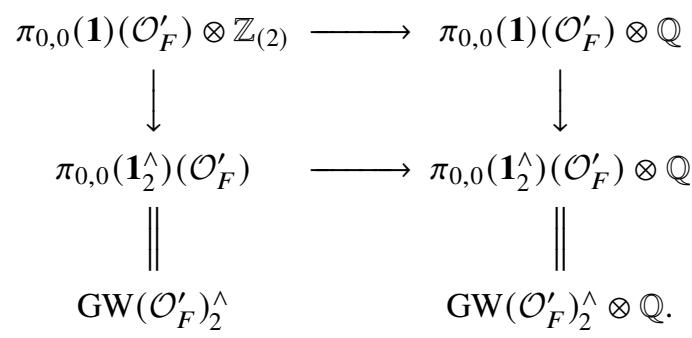

By inspection there are isomorphisms of $\mathbb{Z}\left[\left(\mathcal{O}_{F}^{\prime}\right)^{\times}\right]$-algebras

$$
\begin{aligned}
\pi_{0,0}(\mathbf{1})\left(\mathcal{O}_{F}^{\prime}\right) \otimes \mathbb{Q} & \simeq H_{r e ́ t}^{0}\left(\mathcal{O}_{F}^{\prime}, \mathbb{Q}\right) \times H^{0}\left(\mathcal{O}_{F}^{\prime}, \mathbb{Q}\right) \\
& \simeq \mathbb{Q}^{r} \times \mathbb{Q} \\
& \simeq \mathrm{GW}\left(\mathcal{O}_{F}^{\prime}\right) \otimes \mathbb{Q} .
\end{aligned}
$$

We refer to [7, Theorem 7.2] for a proof of the first isomorphism. Since $\pi_{0,0}(\mathbf{1})\left(\mathcal{O}_{F}^{\prime}\right) \otimes \mathbb{Q}$ is a quotient of $\mathbb{Z}\left[\left(\mathcal{O}_{F}^{\prime}\right)^{\times}\right] \otimes \mathbb{Q}$, in (5.4), the right-hand vertical map

$$
\pi_{0,0}(\mathbf{1})\left(\mathcal{O}_{F}^{\prime}\right) \otimes \mathbb{Q} \simeq \mathrm{GW}\left(\mathcal{O}_{F}^{\prime}\right) \otimes \mathbb{Q} \rightarrow \pi_{0,0}\left(\mathbf{1}_{2}^{\wedge}\right)\left(\mathcal{O}_{F}^{\prime}\right) \otimes \mathbb{Q} \simeq \mathrm{GW}\left(\mathcal{O}_{F}^{\prime}\right)_{2}^{\wedge} \otimes \mathbb{Q}
$$

is the unique $\mathbb{Z}\left[\left(\mathcal{O}_{F}^{\prime}\right)^{\times}\right]$-algebra map. This shows we can identify the square of $\mathbb{Z}\left[\left(\mathcal{O}_{F}^{\prime}\right)^{\times}\right]$-algebras (5.4) with the corresponding fracture square for $\mathrm{GW}\left(\mathcal{O}_{F}^{\prime}\right) \otimes \mathbb{Z}_{(2)}$. It also follows that the unit map to $\mathrm{KO}$ induces an isomorphism, since $\pi_{0,0}\left(\mathrm{KO}_{\mathcal{O}_{F}}\right)=\mathrm{GW}\left(\mathcal{O}_{F}^{\prime}\right)$ is a quotient of $\mathbb{Z}\left[\mathcal{O}_{F}^{\prime \times}\right]$.

Next we show the vanishing $\pi_{*, 0}\left(\mathbf{1}_{\mathcal{O}_{F}^{\prime}}\right) \otimes \mathbb{Z}_{(2)}=0$ for $*<0$. From (5.2), since $a$ is surjective on $\pi_{*}$ and all terms except possibly the top left vanish on $\pi_{*}$ for $*<0$, we deduce that $\pi_{*, 0}\left(\left(\mathbf{1}_{\mathcal{O}_{F}^{\prime}}\right)_{2}^{\wedge}\right)=0$ for $*<0$. Since $\operatorname{GW}\left(\mathcal{O}_{F}^{\prime}\right)$ is finitely generated, the map

$$
\mathrm{GW}\left(\mathcal{O}_{F}^{\prime}\right) \otimes \mathbb{Q} \times \mathrm{GW}\left(\mathcal{O}_{F}^{\prime}\right)_{2}^{\wedge} \rightarrow \mathrm{GW}\left(\mathcal{O}_{F}^{\prime}\right)_{2}^{\wedge} \otimes \mathbb{Q}
$$

is surjective. Considering the fracture square for $\pi_{*, 0}\left(\mathbf{1}_{\mathcal{O}_{F}^{\prime}}\right) \otimes \mathbb{Z}_{(2)}$, it thus remains to prove $\pi_{*, 0}\left(\mathbf{1}_{\mathcal{O}_{F}^{\prime}}\right) \otimes$ $\mathbb{Q}=0$ for $*<0$. This follows from the identification of these groups with subquotients of the rational gamma filtration and rational real étale cohomology, both of which vanish in these degrees, as above.

Applying the same proof method establishes the following odd-primary analog of Theorem 5.2.

Theorem 5.3. Let $\ell$ be an odd prime number. Suppose $F$ is $\ell$-regular and $\mu_{\ell} \subset F$. For the endomorphism ring of $\mathbf{1}_{\mathcal{O}_{F}^{\prime}}$ over the base scheme $\mathcal{O}_{F}^{\prime}:=\mathcal{O}_{F}[1 / \ell]$ there is an isomorphism of $\mathbb{Z}\left[\left(\mathcal{O}_{F}^{\prime}\right)^{\times}\right]$-algebras

$$
\pi_{0,0}\left(\mathbf{1}_{\mathcal{O}_{F}^{\prime}}\right) \otimes \mathbb{Z}_{(\ell)} \simeq \mathrm{GW}\left(\mathcal{O}_{F}^{\prime}\right) \otimes \mathbb{Z}_{(\ell)}
$$

Moreover, we have the vanishing result

$$
\pi_{*, 0}\left(\mathbf{1}_{\mathcal{O}_{F}^{\prime}}\right) \otimes \mathbb{Z}_{(\ell)}=0 \quad \text { for } \quad *<0 .
$$

The same results hold for the motivic sphere over the base scheme $\mathbb{Z}[1 / \ell]$ when $\ell$ is a regular prime.

Acknowledgments. We acknowledge the support of the Centre for Advanced Study at the Norwegian Academy of Science and Letters in Oslo, Norway, which funded and hosted our research project 'Motivic Geometry' during the 2020/21 academic year. We thank the anonymous referees for their valuable comments. This research was supported by grants from the RCN Frontier Research Group Project no. 250399 'Motivic Hopf Equations' and no. 312472 'Equations in Motivic Homotopy’.

Conflict of Interest: None. 


\section{References}

[1] A. Ananyevskiy and A. Druzhinin, 'Rigidity for linear framed presheaves and generalized motivic cohomology theories', Adv. Math. 333 (2018), 423-462.

[2] C. Andradas, L. Bröcker and J. M. Ruiz, Constructible Sets in Real Geometry, Vol. 33 (Springer Science \& Business Media, Berlin, 2012).

[3] M. Artin and B. Mazur, Etale Homotopy, Lecture Notes in Math., Vol. 100 (Springer, Berlin-New York, 1969).

[4] T. Bachmann, 'On the conservativity of the functor assigning to a motivic spectrum its motive', Duke Math. J. 167(8) (2018), 1525-1571.

[5] T. Bachmann, 'Motivic and real étale stable homotopy theory', Compos. Math. 154(5) (2018), 883-917.

[6] T. Bachmann, ' $\boldsymbol{\eta}$-periodic motivic stable homotopy theory over Dedekind domains', Preprint, 2020, arXiv:2006.02086.

[7] T. Bachmann, 'Rigidity in étale motivic stable homotopy theory', Preprint, 2020, arXiv:1810.08028.

[8] T. Bachmann, B. Calmés, F. Déglise, J. Fasel and P. A. Østvær, 'Milnor-Witt motives', Preprint, 2020, arXiv:2004.06634.

[9] T. Bachmann, E. Elmanto and P. A. Østvær, 'Stable motivic invariants are eventually étale local', Preprint, 2020, arXiv:2003.04006.

[10] T. Bachmann and M. J. Hopkins, ' $\eta$-Periodic motivic stable homotopy theory over fields', Preprint, 2020, arXiv:2005.06778.

[11] T. Bachmann and M. Hoyois, 'Norms in motivic homotopy theory', Accepted for publication in Asterisque, Preprint, 2020, arXiv:1711.03061.

[12] T. Bachmann, H. J. Kong, G. Wang and Z. Xu, 'The Chow $t$-structure on motivic spectra', Preprint, 2020, arXiv:2012.02687.

[13] T. Bachmann and K. Wickelgren, ' $A$-Euler classes: six functors formalisms, dualities, integrality and linear subspaces of complete intersections', Preprint, 2020, arXiv:2002.01848.

[14] M. Behrens and J. Shah, ' $C_{2}$-equivariant stable homotopy from real motivic stable homotopy', Preprint, 2019, arXiv: 1908.08378.

[15] A J. Berrick, M. Karoubi and P. A. Østvær, 'Hermitian $K$-theory and 2-regularity for totally real number fields', Math. Ann. 349(1) (2011), 117-159.

[16] A. J. Blumberg, D. Gepner and G. Tabuada, 'A universal characterization of higher algebraic K-theory', Geom. Topol. 17(2) (2013), 733-838.

[17] M. Bökstedt, 'The rational homotopy type of $\Omega$ WhDiff (*)', In Algebraic Topology, Aarhus 1982, Lecture Notes in Math., Vol. 1051 (Springer, Berlin, 1984), 25-37.

[18] A. K. Bousfield, 'The localization of spectra with respect to homology', Topology 18(4) (1979), 257-281.

[19] A. Druzhinin, 'The homomorphism of presheaves $K_{*}^{\mathrm{MW}} \rightarrow \pi_{s}^{*, *}$ over a base', Preprint, 2018, arXiv:1809.00087.

[20] D. Dugger and D. Isaksen, 'Motivic cell structures', Algebr. Geom. Topol. 5 (2005), 615-652.

[21] D. Dugger and D. Isaksen, 'Low-dimensional Milnor-Witt stems over $\mathbb{R}$ ', Ann. K-Theory 2(2) (2016), 175-210.

[22] B. I. Dundas, O. Röndigs and P. A. Østvær, 'Motivic functors', Doc. Math. 8 (2003), 489-525.

[23] W. G. Dwyer and E. M. Friedlander, 'Conjectural calculations of general linear group homology', in Applications of Algebraic K-Theory to Algebraic Geometry and Number Theory (American Mathematical Society, Providence, RI, 1983), (American Mathematical Society), Providence, RI, 135-147.

[24] W. G. Dwyer and E. M. Friedlander, 'Topological models for arithmetic', Topology 33(1) (1994), 1-24.

[25] E. Elmanto, M. Levine, M. Spitzweck and P. A. Østvær, 'Algebraic cobordism and étale cohomology', Preprint, 2017, arXiv: 1711.06258.

[26] E. Elmanto and J. Shah, 'Scheiderer motives and equivariant higher topos theory', Preprint, 2019, arXiv:1912.11557.

[27] E. M. Friedlander, Étale Homotopy of Simplical Schemes, Annals of Mathematics Studies, Vol. 104 (Princeton University Press, Princeton, NJ, 1982).

[28] O. Gabber, 'Affine analog of the proper base change theorem', Isr. J. Math. 87(1-3) (1994), 325-335.

[29] B. Gheorghe, G. Wang and Z. Xu, 'The special fiber of the motivic deformation of the stable homotopy category is algebraic', Preprint, 2018, arXiv:1809.09290.

[30] G. Gras, 'Remarks on $K_{2}$ of number fields', J. Number Theory 23 (1986), 322-335.

[31] G. Gras, Class Field Theory. Monographs in Mathematics (Springer, Berlin, 2003).

[32] G. Gras and J.-F. Jaulent, 'Sur les corps de nombres régulier', Math. Zeitschrift 202 (1989), 343-365.

[33] J. Heller and K. Ormsby, 'The stable Galois correspondence for real closed fields', in New Directions in Homotopy Theory, Contemp. Math. (American Mathematical Society, Providence, RI, 2018), 1-9.

[34] L. Hodgkin and P. A. Østvær, 'The homotopy type of two-regular K-theory', in Categorical Decomposition Techniques in Algebraic Topology, Progress in Math., Vol. 215 (Springer, Basel, 2003), 167-178.

[35] J. Hornbostel and S. Yagunov, 'Rigidity for Henselian local rings and $A^{1}$-representable theories', Math. Zeitschrift 255(2) (2007), 437-449.

[36] M. Hoyois, 'From algebraic cobordism to motivic cohomology', Journal für die reine und angewandte Mathematik (Crelles Journal) 2015(702) (2015), 173-226.

[37] M. Hoyois, 'A quadratic refinement of the Grothendieck-Lefschetz-Verdier trace formula', Algebr. Geom. Topol. 14(6) (2015), 3603-3658.

[38] M. Hoyois, 'Higher Galois theory', J. Pure Appl. Algebra, 222(7) (2018), 1859-1877.

[39] D. Isaksen, Stable Stems. Mem. Amer. Math. Soc. 262 (American Mathematical Society, Providence, RI, 2019). 
[40] D. Isaksen and P. A. Østvær, 'Motivic stable homotopy groups', in Handbook of Homotopy Theory, pages 757-791. Chapman and Hall/CRC, Providence, RI, 2020.

[41] J. A. Jacobson, 'Cohomological invariants for quadratic forms over local rings', Math. Ann. 370(1-2) (2018), 309-329.

[42] J. F. Jardine, 'Motivic symmetric spectra', Doc. Math. 5 (2000), 445-553.

[43] J. I. Kylling, O. Röndigs and P. A. Østvær, 'Hermitian $K$-theory, Dedekind $\zeta$-functions, and quadratic forms over rings of integers in number fields', Camb. J. Math. 8(3) (2020), 505-607.

[44] T.-Y. Lam, The Algebraic Theory of Quadratic Forms. Mathematics Lecture Note Series (W. A. Benjamin, Inc., Reading, Mass, 1973).

[45] M. Levine, 'Convergence of Voevodsky's slice tower', Documenta Math. 18 (2013), 907-941.

[46] M. Levine, 'A comparison of motivic and classical stable homotopy theories', J. Topol. 7(2) (2014), 327-362.

[47] M. Levine, 'Vladimir Voevodsky - an appreciation', Bull. Amer. Math. Soc. (N.S.) 55 (2018), 405-425.

[48] J. Lurie, Higher Topos Theory, Vol. 170 (Princeton University Press, Princeton, NJ, 2009).

[49] J. Lurie, Higher Algebra (2016). URL: http://www.math.harvard.edu/ lurie/papers/HA.pdf.

[50] L. Mantovani, 'Localizations and completions in motivic homotopy theory', Preprint, 2018, arXiv:1810.04134.

[51] A. Mathew, N. Naumann and J. Noel, 'Nilpotence and descent in equivariant stable homotopy theory', Adv. Math. 305 (2017), 994-1084.

[52] J. Milne, Arithmetic Duality Theorems, Vol. 20 (Citeseer, Charleston, SC, 2006).

[53] J. W. Milnor and D. Husemoller, Symmetric Bilinear Forms, Vol. 60 (Springer, New York-Heidelberg, 1973).

[54] F. Morel, 'An introduction to $A^{1}$-homotopy theory', ICTP Trieste Lecture Note Ser. 15, 2003, 357-441.

[55] F. Morel, $A^{1}$-Algebraic Topology over a Field. Lecture Notes in Mathematics (Springer, Berlin, 2012).

[56] J. Neukirch, Algebraic Number Theory, Vol. 322 (Springer Science \& Business Media, Berlin, 2013).

[57] P. A. Østvær, 'Algebraic $K$-theory spectra of non-exceptional two-regular number fields', in Une Degustation Topologique: Homotopy Theory in the Swiss Alps, Contemp. Math., Vol. 265 (American Mathematical Society, Providence, RI, 2000), 187-192.

[58] P. A. Østvær, 'Étale descent for real number fields', Topology 42(1) (2003), 197-225.

[59] P. Pstrągowski, 'Synthetic spectra and the cellular motivic category', Preprint, 2018, arXiv:1803.01804.

[60] J. Rognes and P. A. Østvær, ‘Two-primary algebraic $K$-theory of two-regular number fields', Math. Zeitschrift 233 (2000), 251-263.

[61] O. Röndigs and P. A. Østvær, 'Slices of Hermitian $K$-theory and Milnor's conjecture on quadratic forms', Geom. Topol. 20(2) (2016), 1157-1212.

[62] O. Röndigs, M. Spitzweck and P. A. Østvær, 'Cellularity of Hermitian $K$-theory and Witt theory', in $K$-theory, Proc. Int. Colloquium, Mumbai 2016, Vol. 23 of Tata Institute of Fundamental Research Studies in Mathematics (Hindustan Book Agency, New Delhi, 2018), 35-40.

[63] O. Röndigs, M. Spitzweck and P. A. Østvær, 'The motivic Hopf map solves the homotopy limit problem for $K$-theory', Doc. Math. 23 (2018), 1405-1424.

[64] O. Röndigs, M. Spitzweck and P. A. Østvær, 'The first stable homotopy groups of motivic spheres', Ann. Math. 189(1) (2019), 1-74.

[65] C. Scheiderer, Real and Étale Cohomology, Vol. 1588 of Lecture Notes in Mathematics (Springer, Berlin, 1994).

[66] J. Schmidt and F. Strunk, 'Stable $A^{1}$-connectivity over Dedekind schemes', Ann. K-Theory 3(2) (2018), 331-367.

[67] J.-P. Serre, Local Fields, Vol. 67 (Springer-Verlag, New York-Berlin, 1979). Graduate Texts in Mathematics.

[68] C. L. Siegel, 'Zu zwei Bemerkungen Kummers. Nachrichten der Akademie der Wissenschaften in Göttingen', Math.-Phys. Kl. II 6 (1964), 51-57.

[69] M. Spitzweck, 'A commutative $\mathbb{P}^{1}$-spectrum representing motivic cohomology over Dedekind domains', in Memoires Soc. Math. France (157) (2018), 110 pp.

[70] M. Spitzweck and P. A. Østvær, 'Motivic twisted K-theory', Algebr. Geom. Topol. 12(1) (2012), 565-599.

[71] The Stacks Project Authors, 'Stacks Project', 2018, http://stacks.math.columbia.edu.

[72] V. Voevodsky, 'Open problems in the motivic stable homotopy theory, I', in International Press Conference on Motives, Polylogarithms and Hodge Theory (International Press, Somerville, MA, 2002).

[73] L. Washington, Introduction to Cyclotomic Fields, Graduate Texts in Mathematics, Vol. 83 (Springer Verlag, New York, 1982).

[74] C. Weibel, 'The 2-torsion in the $K$-theory of the integers', Comptes Rendus de l'Académie des Sciences - Series I Mathematics 324(6) (1997), 615-620.

[75] G. M. Wilson and P. A. Østvær, 'Motivic stable stems over finite fields', Algebr. Geom. Topol. 17(2) (2017), $1059-1104$. 